\title{
The Theory of Elementary Waves
}

\author{
Lewis E. Little
}

\begin{abstract}
A fundamental error is identified in the foundations of current quantum theory. The error is shown to be the source of the various noncausal and unphysical aspects of the theory. When the error is corrected, a new theory arises, which is both local and deterministic, but which nonetheless does not conflict with Bell's theorem. The new theory reproduces quantitatively all the predictions of current quantum mechanics, with the exception of double-delayed-choice Einstein-Podolsky-Rosen (EPR) phenomena. A shortcoming in Aspect's experiment testing such phenomena is pointed out, and a definitive experiment is proposed. The EPR paradox is resolved. The uncertainty principle is derived on a causal, deterministic basis. The theory is "automatically" relativistic; that is, the constancy of the velocity of light $\mathrm{c}$ relative to all observers follows as an immediate consequence of the new quantum theory, which constancy thus acquires a simple physical explanation. General relativity also acquires a simple, physical explanation. A pictorial interpretation of Feynman diagrams is obtained. The theory provides a clear physical explanation for the Aharonov-Bohm effect and suggests an explanation for the irreversibility of quantum statistical processes. Quantum statistics, Bose and Fermi, are explained in a simple, pictorial manner. Overall, it is shown that quantum and relativistic phenomena can be understood in an objective manner, in which facts are facts, causality is valid, and reality is real. The theory provides a single framework in which all known physical phenomena can be comprehended, thus accomplishing the objective of a unified field theory.
\end{abstract}

Key words: quantum theory foundations, Bell's theorem, delayed choice, special relativity, Feynman diagrams, Aharonov-Bohm effect, general relativity, unified theory, reality

\section{INTRODUCTION}

Most physicists today believe that a local, deterministic theory of quantum phenomena is impossible. Bell's theorem, ${ }^{(1)}$ backed up by various experiments ${ }^{(2,3)}$ of the Einstein-Podolsky-Rosen ${ }^{(4)}$ (EPR) variety, is generally taken as proving this conclusion. Most notably, the experiment of Aspect $^{(5)}$ is viewed as a clear observation of nonlocal behavior.

It will be demonstrated in this paper that the inability to explain quantum phenomena in a local, deterministic manner is not the product of a nonlocal, noncausal universe. Rather, the inability stems from a single basic physical error made in the early days of quantum theory, which error produced all the myriad contradictions that make up the "weirdness" of current quantum mechanics. When corrected, a very simple, causal, local theory immediately appears.

Mathematically the new theory is, at the level of Schrödinger waves and their matrix elements, identical in most respects to current quantum mechanics. The underlying foundations of the theory are greatly simplified, but the mathematical expressions for the matrix elements for all single-particle processes remain unchanged.

It might be thought that if the mathematics is the same then the theory must be the same. The nonlocality and indeterminism of current theory are generally viewed as being part and parcel of the mathematics. That this is not the case - that the various "weirdnesses" are the product of unidentified (and incorrect) physical assumptions and are not inherent in the mathematics - will be demonstrated by producing an actual theory that is local and deterministic, but without changing (most of) the (matrix element level) mathematics.

The proposed theory is not a hidden-variables theory in the sense of a theory that accepts present quantum mechanics and then adds new variables. Rather, current theory is modified by correcting the basic physical error, with the result that one can understand the mathematics in a local, deterministic manner without the addition of any new variables. In the new form, however, it will be shown that one can account for the unpredictability - as opposed to indeterminism - of particle behavior as being the product of "hidden" variables that have exact - if unknown - values at all times.

\section{PRELIMINARY EXPERIMENTAL EVIDENCE}

To begin presenting the evidence for the proposed theory, and for the error in present theory, I will take a new look at a number of the key experiments confirming quantum behavior. Some of these experiments were not available when quantum mechanics was first developed. Had they been available, some very different theoretical conclusions might have been drawn. So, how might the experimental evidence available today be interpreted using the principles generally accepted in the prequantum era?

By "prequantum" principles I do not mean "classical" physics per se. Much about the proposed theory will be very nonclassical. Rather, I mean simply that the experiments are to be interpreted 
based on the view that real objects have a single identity: that a wave is a wave and a particle is a particle, that a particle is located in one place at one time and not many places simultaneously, etc. Facts are facts. Cause and effect is strictly obeyed.

And, in particular, nonlocality is unacceptable in this view of things. Nonlocality implies that distant events can affect one another instantaneously by no physical means. But no effect can be produced by no means. Nor can any effect be propagated over a distance instantaneously, which would imply an absence of means as well as contradicting the well-established fact that physical effects cannot propagate with a velocity greater than $c$, the velocity of light.

Consider first the standard double-slit experiment. This is the basic experiment confirming the wavelike behavior of particles. One sees a wavelike pattern on the screen but only as the result of numerous individual particle events. Each particle is observed to arrive at only one point on the screen.

If one tries to observe particles at any point before or after the slits, or as a particle passes through a slit, one always observes only particles - with a single location - never a wave. Nonetheless, a wavelike pattern appears on the screen (assuming no attempt to observe the particles before they arrive at the screen). So clearly both waves and particles are present. One sees waves and one sees particles, so one has both waves and particles.

Yet, as is well known, all attempts to interpret the experiment using separate waves and particles have failed.

To begin to see why, consider the following additional fact about the experiment. Suppose one tries to explain the pattern on the screen with a hypothetical set of particle trajectories. The maxima on the screen might then be explained by particles following the trajectories shown in Fig. 1. However, if the screen is moved to position B, clearly the particles from each slit, and still following those same trajectories, will no longer arrive at the same points on the screen; the particles from one slit will fall somewhere between the points of impact of the particles from the other slit. The pattern would then be washed out. And yet a similar wave pattern is observed at all screen distances.

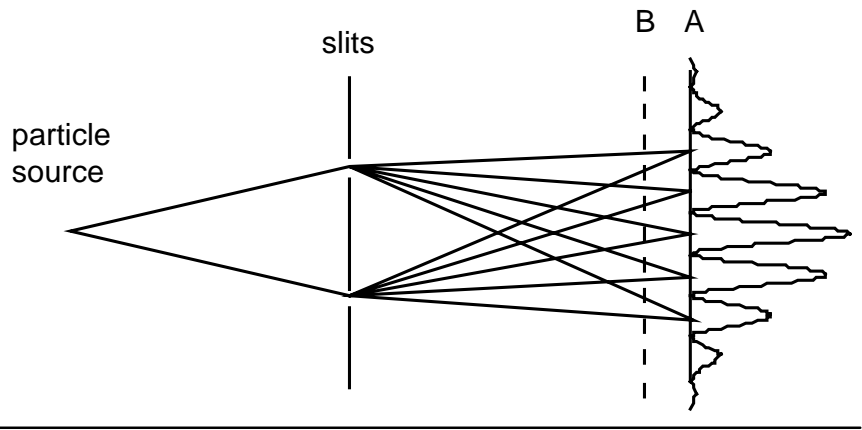

Figure 1. Double-slit experiment with hypothetical trajectories

If the particles are assumed to be particles, and if they follow straight lines between the slits and the screen, there is only one conclusion that can be drawn: the trajectories depend on the screen position. If one moves the screen the particles follow different trajectories.
But this could only happen if something is moving from the screen to the oncoming particles to affect their motion. Without some real, physical process to explain the screen dependence, one would be left with the need for a nonlocal interaction to account for the dependence. If one rejects nonlocality, then this experiment constitutes direct observational evidence of the reverse motion of something from the screen to the particles.

Perhaps the reader is so used to the usual quantum mechanical description of this phenomenon, in which the particles follow no trajectory in particular, that it is not immediately apparent that the trajectories are screen-location-dependent. But unless one is already wedded to the usual quantum picture, what one has here is a direct experimental observation of the fact that something moves from the screen to the particles. There is no other local manner in which one can explain what is observed.

One might try to invent a theory such as Bohm's ${ }^{(6)}$ in which a potential of some kind exists in the region behind the slits. This potential would not depend on the screen position, but rather only on the slits. The particles would then follow curved paths of some kind, but paths that do not change as the screen is moved. However, as proved indirectly by Bell's theorem, it is impossible to accomplish this with a local potential. And Bohm's "quantum potential" is explicitly nonlocal.

Something has to move from the screen to the particles.

Consider next the emission of photons by an excited atom in a resonant microcavity. Experiment ${ }^{(7)}$ confirms that the atom can emit a photon only if the appropriate state is available in the cavity - as this is described in current theory. If the cavity is "mistuned," the atom cannot radiate.

Clearly, the cavity affects the emission process. But this can only happen if something travels from the cavity to the atom to affect its emission. Otherwise, there must be a nonlocal interaction with the cavity walls, or the photon must first be emitted and then unemitted if the necessary state is found to be unavailable. Neither alternative makes any sense. Again, we see the reverse motion of something.

Perhaps the best example confirming the reverse motion is provided by EPR experiments. Consider, to be specific, the experiments with two photons and measurements of their polarization. ${ }^{(2)}$ Bell's theorem, ${ }^{(1)}$ generalized to this experiment, ${ }^{(8)}$ proves - although it is not usually interpreted in this manner that whatever variables might describe the photon on one side of the experiment, there must be a dependence on the orientation of the polarizer on the other side. Otherwise, one cannot explain the observed correlations between the polarizations of the two photons. This must be true for any description of the photon based on parameters that have exact values. But given our "prequantal" outlook, all parameters must have exact values, and nonlocality is unacceptable. Hence what we have is a direct proof that the photon state depends on the orientation of the opposite polarizer.

It might seem strange to interpret Bell's theorem as proving the fact of this dependence. Bell's reasoning was premised on the absence of any such dependence. He then proved that given this lack of dependence, there is no local manner in which one can account for what is observed, assuming a description of the photons based on parameters with exact values. But far from proving nonlocality and/or the absence of parameters with exact 
values, as this is frequently interpreted, the fact that nonlocality and the absence of exact parameters are unacceptable means instead that the theorem is a reductio ad absurdum of its major premise. The photon state must depend on the polarizers, because if not, one is forced to accept nonlocality and/or the absence of parameters with exact values describing the photons. But both of these latter conclusions are absurd.

But, again, this dependence could only occur if something travels from the polarizers to the photons and/or the photon source to cause the dependence. Again we see evidence - in this instance proof - that something moves in reverse.

But what about Aspect's experiment ${ }^{(5)}$ with "double-delayedchoice"? It would seem that even with something traveling from the polarizers toward the photons there would be no way to account for his result locally. When both polarizers are rotated in the delayed manner, there isn't time for a signal from one polarizer to reach the photon on the other side before it reaches its polarizer.

As will be demonstrated in Sec. 4, there is a shortcoming in Aspect's experiment, arising from the repetitive switching back and forth between the same two polarizer states on each side of the apparatus. For the parameters chosen by Aspect, something traveling in reverse, from polarizers to photons, will, in fact, explain his result in a local manner. A proposal for a definitive experiment that corrects the shortcoming will be made in Sec. 4. A local explanation for the single-delayed-choice experiments will also be presented.

All instances of noncommuting observables constitute similar evidence of reverse motion. The state of a particle depends on the measuring device one uses to observe it. If one uses one measuring device, what is observed contradicts anything that might have been observed with another ("noncommuting," so to speak) device. Indeed, the observation with one device forces one to conclude that the particle had no state in particular for the other device, an absurd conclusion, if, again, we are maintaining that facts are facts.

But the state of a particle can only depend on the measuring device if something moves from the device to the oncoming particle to affect its state. There must be a real physical basis for the dependence.

So if looked at "prequantally," the collective experiments yield much evidence of something moving in reverse - from a detector or measuring device to the particles that will be observed by that device. There is no other local manner in which one can explain what is observed.

But at the same time we have the mountainous evidence that the equations of current quantum mechanics work. When applied to any known physical system, those equations yield what is observed in the laboratory. Yet nothing moves in reverse in current theory. If some new entity is to be added, moving in reverse, how can one still explain the fact that the current mathematics works so well?

The essence of the proposed answer is very simple: it is the quantum wave itself that moves in reverse. With a reverse wave all that changes mathematically is the sign of the momentum exponent in the exponential describing the wave. (It will be shown later that even that change is not necessary.) But that exponential is squared in absolute value when deriving an observable result anyway; so a change to its sign changes nothing. And by reciprocity ${ }^{(9)}$ the matrix element for any scattering of a reversed wave is identical, but for a possible phase factor, with the forward scattering. If one can make a theory work with reverse waves, it should yield the same mathematics and yet still provide the "something" that moves in reverse.

It must be the quantum waves that move in reverse. We know by direct observation that the particles move forward; and we know, with near certainty, if not complete certainty, from the mathematics of the current theory that nothing other than the particles and the quantum waves is involved. It is difficult to imagine how some third thing might be involved, and yet still permit one to recover the current mathematics. So the only thing left to move in reverse is the quantum wave itself.

Clearly, reverse waves imply a radically different theory. No longer are the waves somehow the particles. Rather, the waves are present in the environment already, and the particles then follow those waves. But enough evidence supports such a picture to warrant its consideration.

The reason for the failure of all previous attempts at a theory with separate waves and particles - indeed, of all previous attempts to account for quantum behavior in a local, deterministic manner - is that the waves were always assumed to move forward, with (or as) the particles. Because the waves actually move in reverse, as will be even more fully demonstrated in what follows, and carry with them "information" regarding the environment into which a particle is moving, all such forwardwaves theories were necessarily nonlocal. The physical effects caused by the "information" carried by the reverse waves could only be accounted for through one kind or another of nonlocal interaction. This, I submit, is the real physical basis for Bell's theorem.

The basic error in current theory is that the waves are moving in the wrong direction.

\section{OUTLINE OF THE THEORY}

Consider how the reverse waves might explain the double-slit experiment. Imagine that every point on the screen is continually emitting waves, having the same properties as the usual quantum waves. The waves in all directions from a single point on the screen are mutually coherent; but the waves from different points are mutually incoherent. The waves from a given point penetrate back through the slits, and the two wavelets leaving the slits toward the particle source then interfere with one another. Clearly, by reciprocity, the waves from a point on the screen corresponding to a "light" fringe (many particles reaching this point) would interfere constructively at the particle source. Waves originating from a "dark" fringe (no particles) would interfere destructively. Waves from an intermediate point would suffer partial destructive interference. Indeed, by reciprocity, the intensity of the wave reaching the source from a point on the screen is identical to the intensity of the usual forward-moving quantum wave reaching that point on the screen, assuming identical intensity upon emission.

Suppose further that a particle is emitted by the source only in response to the stimulation of these waves, with the probability of emission being proportional to the intensity - the absolute value 
of the amplitude squared - of the waves. Suppose further that the particles, once emitted in response to the wave from a particular point on the screen, are causally determined to follow that wave to that point on the screen.

So particles reach the "light" fringes because the coherent intensity of the waves from those screen locations is a maximum at the particle source, and many particles are created in response to those waves. No particles reach the dark fringes because the waves from those fringes suffer destructive interference after penetrating the slits, and no particles are generated.

At every point on the screen the number of particles arriving is proportional to the intensity, at the particle source, of the wave emitted by that point. But, again, that intensity is the same as the intensity at that point on the screen of the usual, forward-moving wave, assuming equal intensity upon emission. And that latter intensity gives, in current theory, the probability that a particle is observed. So, if one can explain how the screen emits the waves and how the particles then follow them, this picture would account for what is observed on the screen in exact mathematical detail.

The wave is present at all times and not only when the particle is emitted. There is thus no problem in explaining why the wave is present when the particle "needs" it. And the wave does not have to "carry" the particle in any sense. The particle simply follows the direction from which the wave is coming (by a process that will be described in detail in Secs. 8 and 9), following it back to its source, which it reaches with probability 1 . No nonlocal interaction between an extended wave and the particle is required to understand how the particle follows the wave. The theory is both local and deterministic. Waves are waves and particles are particles, and both have an exact state at all times.

Because the particle follows the wave, the physics of the particle motion is determined entirely by the wave, which is why the wave computation determines the ultimate trajectory or set of trajectories. But it is the intensity of the wave at the source - the amplitude squared - that gives the probability that the particle is created to follow that particular wave. Thus we see in trivial physical fashion why one computes wave amplitudes and then squares to get the probability of the particle process. Rather than yielding the probability that a particle is somehow "created" at the screen out of the wave, the square gives the probability that a particle is created at the source to follow that particular wave in the first place. The square occurs at the source of the particle, not at the detector.

The particle might follow any one of many paths "through" the wave; but any one particle follows only one path. It makes no difference to the cross section - the probability that a particle reaches the particular point on the screen - which path the particle takes. The cross section is already determined at the particle source by the intensity of the wave reaching the source. All that is necessary to reproduce what is observed is that the particle, once emitted in response to the particular wave, reach the source of the wave at the screen by some path.

The particle travels through only one slit. The wave goes through both slits. But the wave goes through first, setting up the interferences, before the particle arrives.

With this picture one does not even need any "measurement theory" to understand what happens when the particle reaches the screen. The squaring of the wave takes place at the particle source; and this makes perfect sense: one would expect the probability of particle emission to be proportional to the intensity of the stimulating wave. At the screen one simply sees the particle with probability 1. There is no wave function "collapse"; the wave is there all the time.

As discussed by Feynman, ${ }^{(10)}$ the double-slit experiment captures the entire essence of the "problem" of quantum mechanics. A theory that can account for this experiment in a local, deterministic manner should be able to account for all quantum phenomena in a similar manner.

Even if the picture sketched here is found to be incorrect in the light of evidence from other experiments, the picture nonetheless provides, in principle, an explanation for the double slit that is both local and deterministic. But according to the currently accepted view, this should be impossible. Clearly, by explicit example, it is possible. The various alleged proofs to the contrary all make, implicitly or explicitly, physical assumptions, in particular, the assumed forward motion of the waves. With changed assumptions the proofs are no longer applicable.

The prescription outlined here for the double-slit experiment works immediately for any experiment in which particles are emitted by a source, penetrate through or scatter from a system of some kind, and are then observed at a detector. Each point on the detector emits waves, just as with the screen above. Those waves penetrate back through the system. By reciprocity, which applies generally to any kind of system, the intensity of the wave at the particle source is the same as the intensity of the usual, forwardmoving quantum wave at the same point on the detector, assuming equal emission intensity. So if particles are created at the source in proportion to the intensity of the reverse wave, and if those particles then follow that wave to the detector with probability 1 , the probability of seeing the particle at the given point on the detector is exactly the same as in current theory.

So we see with complete generality why one adds amplitudes and squares to get a probability. We also see why it is the wave computation that yields the particle trajectories.

If no detector is present at a point along the path of the particles, then no reverse waves are emitted at that point. Rather, the waves originate from another detector or object further "downstream," downstream, that is, from the point of view of the particles. So the dynamics of the wave at the given location when the detector is absent is determined by the first power of the wave function; the first power is what appears in the Schrödinger equation. But if one inserts a detector, the reverse waves now originate from that detector. So the probability of detecting a particle is given by the square - at the source - of the wave. But the square at the source of the reverse wave is the same as the square at the detector of the usual quantum wave. So we see why the first power determines what is present when one doesn't look, but the square gives what one sees when one does look

And, just as for the double slit, if a particle might take more than one path between the source and the detector, any interference is explained by the fact that the reverse waves take all paths. Each particle takes only one path. It is never necessary to have a particle in more than one location at a single time. Instead of particles being two places at once, one simply has two waves. 
Perhaps the best feature of this picture is, again, that it requires no special measurement theory. When a particle arrives at a detector, it is simply observed with probability 1 (assuming perfect detector efficiency). There is no wave function "collapse," no transition from microscopic to macroscopic, or what have you. The wave needn't (nonlocally) disappear in order to prevent the generation of two or more particles by a single-particle wave, as in current theory. The wave simply remains, stimulating further particles from the source as long as the experiment lasts. It makes no difference that the wave leaves the system in directions other than the source, because there are no sources in other directions. Or if there were other sources, one would expect other particles.

We thus immediately have a theory that will account — locally and deterministically - for all single-particle experiments in which the apparatus through which the particle moves is static. (In dynamic systems the reverse waves will change before the particle arrives.) This includes the vast majority of quantum experiments.

And this has been achieved with virtually no change to the mathematics and, in particular, with no additional variables, "hidden" or otherwise. The cross section for any process is determined by the reverse wave matrix elements, which are equal to the forward-wave matrix elements but for a possible phase factor. All that is necessary to make perfect sense out of the current mathematics is simply to reverse the direction of the waves.

Schrödinger's cat paradox ${ }^{(11)}$ receives a trivial resolution in this theory. If a particle is going to change from one state to another, as in the decay of a radioactive nucleus, the waves for both states exist and interact throughout the process. There is never any need to assume that the particle itself is in both states simultaneously. The decisive moment, when the square is computed, is not at the point of observation, but rather at the point of emission. The act of observation itself - looking at the dial (or in this case the cat) as opposed to inserting the detector - plays no role.

The reverse wave picture immediately explains the phenomena associated with noncommuting observables. One cannot measure two such observables because one cannot simultaneously set up the reverse waves corresponding to both. The apparatus and/or detectors that would yield the waves for one variable destroy the waves corresponding to the other.

In current quantum mechanics the value of a measured parameter cannot be viewed as existing prior to a measurement. In some way the act of measurement puts the system into the state measured. The reverse waves theory shows that this latter idea is, if anything, an understatement. The act of measurement affects the very creation of the particle in the first place. (It will be shown later that the particle "creation" that is relevant might actually occur close to the measuring apparatus and not at some distant particle source.) The particle that comes into existence at the source is determined in its state in part by the reverse wave, which wave depends in turn on the experimental apparatus employed. But now the sequence of events by which the act of measurement affects the particle makes sense. In current theory the effect of the measurement on the particle occurs at the detector. A mysterious, noncausal, and nonlocal jump into the state determined by the act of measurement must take place, and prior to this the particle has (in general) no value in particular of the measured parameter. With the reverse waves the effect occurs at the source. Waves exist corresponding to all possible values of the parameter, and so the emitted particle might have any one of these values. But once created with a particular value of the parameter, the particle maintains that value at all times. There is thus no contradiction between the conclusion that the act of measurement affects the value measured, while simultaneously maintaining that the value of the parameter exists prior to the measurement, prior, that is, to the actual detection of a particle at the detector. There is no unknowable "jump" upon detection.

Consider the experiment with the atom in the resonant microcavity. The explanation of how the cavity affects the emission is that the wave is emitted by the cavity. All different frequencies of waves are emitted by the cavity walls, but only those corresponding to resonance of the cavity will interfere constructively and remain in the cavity. Other frequencies suffer destructive interference as they reflect back and forth in the cavity. A photon will only be emitted if the wave of the proper frequency is present to stimulate the emission.

Notice that if the cavity is "tuned" to the atom so that a photon can be emitted, then the "available state" into which the photon is emitted - as described in current theory - is, of course, mathematically identical to the photon wave itself when it is emitted. What the reverse wave theory proposes is that these available state waves are, in fact, real reverse waves emitted by the cavity. So, instead of having the wave emitted as the photon, the photon is simply a particle emitted in response to the already existing "available state" wave. The particle photon then follows that wave. The only change is that the wave moves in reverse, from the cavity walls to the particle photon source.

Mathematically, again, this is identical to current theory. The matrix element for the emission is identical. All that we have done is to say that the exponential factor in the matrix element corresponding to the emitted photon wave (in current theory) is instead the available state, reverse wave which stimulates the emission. We have simply changed the physical interpretation of the same mathematical expression. But now the causality of the process makes sense. The "weirdness" has been eliminated, but with no change to the mathematics.

Indeed, all particle emission, as described by current theory, requires the availability of a final state. But how can the mere availability of a state affect the emission process? In order for the available state to affect something, that state must be something itself. The mere "place" where something might go isn't anything in itself. So the very fact that the quantum description works and requires an available state serves as evidence that those available states are, in fact, something in their own right - something real - that are present in the environment before a particle is emitted.

And those available states, in order that they be able to affect an emitting system, must move toward that system, that is, in the direction opposite to that in which the particle will move when it is emitted. Any phenomenon involving an available state thus constitutes further evidence of the reverse motion of something.

Because all final state particles in any interaction require the availability of a final state, and because all initial particles were themselves final particles in some previous interaction, this 
prescription should work generally for all particle processes. Whatever the available final state is in current theory, simply reverse its direction, say that that wave stimulates the emission of the particle and that the particle then follows that already existing wave. It will be shown in Sec. 8 that this prescription works in general to explain Feynman diagrams in a straightforward, pictorial manner.

It is clear qualitatively how the reverse waves picture will explain EPR experiments, or at least those without delayed choice. The reverse waves penetrate the polarizers before they arrive at the particle source and thus carry with them "information" regarding the polarizer orientations. The particle photons are then created in a state that reflects the polarizer orientations at the outset. Bell's major premise is violated: the variables that describe the photons do depend on both polarizer orientations. (EPR will be treated quantitatively in Sec. 4.)

I have spoken of the reverse waves as being "emitted" by the detector. This is not strictly correct. Particles can, of course, be emitted by a source into free space and not simply in the direction of a physical detector. So if particle emission requires an available, reverse wave, the reverse waves corresponding to free-particle states must exist also. We must have reverse waves corresponding to all possible particle states. That is, waves exist corresponding to a complete set of quantum states. All such wave states must be filled by a wave, whether or not a physical detector is present to emit them. The postulate, then, is that a complete set of waves exists at all times.

It may seem far-fetched to postulate the existence of such a complete set of waves, moving in all directions, with all frequencies, corresponding to all kinds of particles. However, this picture is not substantially different from the usual classical picture of a lighted room. Electromagnetic waves of all frequencies albeit with amplitudes that vary with frequency - and moving in all directions, fill the room. This is, in essence, the picture I propose for the reverse waves.

In the theory that I will develop through the remainder of this paper, these waves exist independently, in addition to the elementary particles. I will argue that they are primary constituents of reality on the same level as the elementary particles. In particular, they are not waves in any kind of medium. For this reason I will from now on call them "elementary waves."

The elementary waves are real waves. They are not simply a mathematical fiction allowing one to obtain the correct answer for the particle process or the like. They exist as real objects.

Because the waves are not waves in a medium, they do not propagate according to the usual dynamics of waves. In fact, as will be described more fully later, the description of their propagation is much simpler than that of the usual waves. They actually propagate much like a simple flux of material, with the material carrying a wave "implanted" in it, so to speak. However, the product looks exactly like a wave propagating according to the usual field equations.

Detectors and other particulate objects do not actually emit these waves. The waves are present continually and with constant intensity. All that detectors - particles or combinations of particles in general - do is to establish mutual coherence among the waves leaving their vicinity. An organization is imposed on the already existing waves. It is the mutual coherence that then leads to the observed interference effects. I will continue to refer to detectors as "emitting" the waves, but this must be understood in this sense.

The quantity of wave material along any direction in space never changes, even in a "scattering" of the wave. All that happens at a scattering vertex is that the coherence of the incident wave flux becomes rearranged due to the interaction with the other waves at that vertex. When two waves interact, one wave might impose its coherence on the other. This gives the appearance that the second wave is the product of a scattering of the first wave; but in fact no actual scattering occurs.

The processes by which the coherence is imposed by a detector will be discussed in Sec. 9. But clearly the wave processes involved must correspond to inelastic particle processes. It is only by inelastic processes that we observe particles. So at the detector, wave processes occur looking exactly like the wave process at the detector in current theory, but in reverse. When a particle arrives at the detector while following the resulting wave, the particle continues to follow the wave as it scatters; the particle "mimics" the wave process in reverse. But it is specifically the inelastic processes that are relevant to a detector.

While the total wave intensity in any single-particle state is a constant, the wave can be divided into separately coherent "pieces." A wave state can act as if it were empty by having its "pieces" arranged to be mutually coherent but out of phase with one another. This is what occurs in the resonant microcavity for the "mistuned" states.

The theory requires that the separate, mutually incoherent "pieces" of a single wave state act independently from one another, so one adds intensities at a particle source, not amplitudes. Also, "pieces" can be mutually coherent while still having different phases: one adds amplitudes, not intensities. Hence waves that are mutually coherent must be able to "recognize" one another, and waves that are not mutually coherent must also be able to recognize this fact. How this occurs will be discussed in Sec. 15.

All the dynamics of particles are determined by the waves. The particle itself needn't carry any of the "classical" dynamic quantities generally attributed to particles: mass, momentum, energy, etc. All these properties describe only the waves, with the particle then acting accordingly. Particles need carry only those parameters required for them to recognize and follow their wave. Of course, it is by virtue of these latter parameters that a particle will follow only waves of particular characteristics; so in this sense one might say that the particle has mass or momentum or what have you. But the actual numerical quantity is carried by the wave. This, of course, accords directly with the mathematical description of "wave-particle" dynamics in current quantum theory. I will continue to describe a particle as having momentum or energy or etc., but this must be understood simply as meaning that it is following a wave with these characteristics.

Particles are emitted in response to waves of particular frequency/momentum. The behavior of the particle then reflects exactly the momentum of the wave. There is no "uncertainty" in the emission process. The process does not follow a "classical" model, in which the source "measures" the frequency of the wave 
and then emits a particle of appropriate momentum. In this latter model the momentum of the particle would be uncertain, given the finite time period during which the source would "measure" the wave's frequency. No such uncertainties are involved here.

Because the waves exist in their own right, there is no need to somehow obtain the laws of the waves from those of the particles, as is done in the usual canonical quantization procedure. It is from the observed behavior of particles that one determines the fact that the waves exist and what their properties are; but once one knows their properties, one simply says that the waves exist. There is no need to explain their properties from something else. Canonical quantization becomes entirely superfluous in this theory.

The full mathematics of the waves will be developed in Secs. 7 through 9. However, with the above partial picture one can deduce a few more quantitative results of some consequence.

\section{EINSTEIN-PODOLSKY-ROSEN PARADOX}

The elementary waves theory yields a quantitative resolution of the EPR paradox. ${ }^{(4)}$ Consider again the experiments with photons, in particular, the experiment of Freedman and Clauser, ${ }^{(2)}$ pictured in Fig. 2. An atom decays twice in a $J=0 \rightarrow J=1 \rightarrow J=0$ cascade, emitting two correlated photons in opposite directions, which then traverse polarizers and, if not absorbed in the polarizers, strike detectors. If the two polarizers are orientated an angle $\theta$ apart, quantum mechanics predicts a $\cos ^{2} \theta$ dependence for observing coincidences (assuming perfect polarizers and detectors), a dependence thought not to be explainable in a local, deterministic manner. ${ }^{(8)}$

In the elementary waves theory waves are "emitted" by both the detector and the polarizer on both sides of the experiment. The detector emits waves of all polarizations. However, as these waves penetrate the polarizer, half are absorbed and the other half become polarized parallel to the polarizer's axis of transmission. In addition, the polarizer itself emits waves. Just as it absorbs photons that are polarized perpendicular to its axis of transmission, it emits only such waves. (An object "emits" only those waves that correspond to particles that it would absorb, for reasons to be explained more fully below.) So two waves arrive at the photon source: the polarized wave from the detector and the perpendicularly polarized wave from the polarizer.

(Actually, however, what happens is a little more complicated. None of the elementary waves are actually absorbed; all states, again, are always full. What the polarizer does is impose mutual coherence among waves with equal and opposite polarization angles, so that the pair acts as a unit, polarized either parallel or perpendicular to the polarizer axis. The polarizer emits only such pairs polarized perpendicular to the transmission axis. It transmits only such pairs polarized parallel to the axis.

The details on how a polarizer accomplishes this will not be presented explicitly in this paper. However, once the mathematical equivalence between the elementary waves theory and current quantum mechanics is established in Sec. 9, it will be clear that the process can be understood by direct parallel to the current explanation of polarizer action.)

When the waves arrive at the photon source, they stimulate the emission of photons. The two waves - one from the polarizer and one from the detector - are not mutually coherent because they arise from different sources; so they act independently in stimulating the emissions. When a photon is emitted in response to one of the two polarized waves, it follows that wave to its source - either the polarizer or the detector — with probability 1 . No waves coming from the direction of the polarizer are present other than these two, so only photons following one of these two waves are emitted toward the polarizer.

Suppose the two polarizers are orientated an angle $\theta$ apart, and the decaying atom is stimulated to emit the first photon in response to the wave that traverses its polarizer from the detector, which photon will thus itself traverse the polarizer and be detected. Now the atom wants to emit a second photon in the

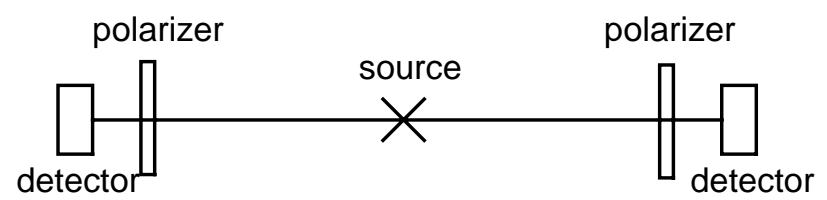

Figure 2. EPR experiment of Freedman and Clauser.

opposite direction with the same polarization as the first. But there is no stimulating photon elementary wave with this polarization, only one an angle $\theta$ apart, coming from the detector on the other side and another an angle $\theta+90^{\circ}$ apart coming from the polarizer. Each of these waves might stimulate the emission of the second photon by the atom, but with a diminished probability. The amplitude of the first wave, relative to the needed polarization, is $\cos \theta$, so the probability - proportional to the intensity of the stimulating wave - goes as $\cos ^{2} \theta$. This gives the probability that the second photon will be emitted in response to the wave that traverses the other polarizer and hence the probability that the particle photon will do the same, and be detected. So the probability of coincidence is exactly the result predicted by quantum mechanics. The stimulating photon elementary wave at angle $\theta+90^{\circ}$ will, with probability $\sin ^{2} \theta$, create a photon which is then absorbed in the polarizer.

The key to making sense out of the $\cos ^{2} \theta$ dependence is that the square occurs at the source, which in turn results from the reverse motion of the waves.

To be strictly accurate, it isn't the case that the atom emits two photons in separate processes. As will be explained more fully in Sec. 12 , the cascade is actually a single quantum process for which a single overall amplitude needs to be computed. An overall wave interaction, involving both photon elementary waves, occurs before either particle photon is emitted. Current theory obscures this point, because it makes the particle into the wave. Thus the electron waves corresponding to the middle and lower level in the emitting atom do not exist until the jump into those levels occurs. There is no way that the interactions corresponding to the emission of the second photon can begin until the first photon has been emitted. But in the elementary waves theory the waves for all three levels exist at all times, and the interactions corresponding to the cascade thus also exist at all times. Whatever cascade occurs, the corresponding overall wave interactions were taking place prior to 
the emission of the first photon. If the photon waves have polarizations that are orientated an angle $\theta$ apart, then the amplitude goes as $\cos \theta$. Hence the probability of the two-particle process goes as $\cos ^{2} \theta$. The above two-step description, although inaccurate, is offered here to help visualize the origin of the $\cos ^{2} \theta$ factor.

Current theory is actually inconsistent on this point. If the two steps in the cascade were actually independent, then the resulting photon waves would not be mutually coherent/entangled. To be entangled, as per current theory, one must have a single amplitude. But there is no mechanism for this if the particle is the wave. The mathematics can be made to "work," but the theory is inconsistent.

Notice, however, that the photon waves on either side of the photon source in the above elementary waves explanation for EPR are not in any way "entangled." Each wave is simply a plane wave (approximately) with phase determined solely by the detector or polarizer from which it originated. The effects of the two waves at the source are, one might say, "entangled," that is, the emission of each photon is affected by both waves, but not the waves themselves.

Entangled wave functions are necessary in current theory because of the forward motion of the waves. The actual "entanglement" occurs at the photon source, as just indicated. But that entanglement must, mathematically, be present at the location where the square is performed. With forward-moving waves the squares occur at the polarizers and detectors, not at the source. So in order to make the forward-wave theory work, the waves must be entangled - with subsequent "collapse" - in order to carry the entanglement from the source to the detectors. With reverse waves no wave entanglement is necessary. Each wave is simply an independent, single-particle wave. As will be demonstrated in Sec. 11, quantum statistics in general can be accounted for without wave entanglements.

Wave entanglements are generally viewed as being essential to the description of identical particle phenomena and to the entire structure of quantum mechanics; so it may strike the reader as absurd to try to account for multiparticle effects without them. But certainly the above EPR experiment is one instance where the effects in question are manifested. And, using reverse waves, as just demonstrated, the correct result is obtained with no entanglements. It really is only the erroneous forward-wave motion that gives rise to them.

The elimination of wave entanglements constitutes the only major change to the mathematical formalism (at the matrix element level) of quantum mechanics that is required by the elementary waves theory. But clearly this change represents a major simplification. In general, no multiparticle states are necessary in the theory.

Such multiparticle states are, or course, nonlocal in their behavior. One would thus expect them to disappear in a local theory.

Actually, the independence of the elementary waves from different detectors or from different points on a detector is a general property of the theory, even for single-particle phenomena, as indicated above. In current theory the wave arriving at various points of a detector, even for a single-particle wave, must be treated as a single coherent wave. It is the self-interference of this single wave from the source that produces the various quantum wave effects. That wave then "collapses" (nonlocally) when the particle is observed. In the elementary waves theory the interference occurs in the reverse direction; the wave from each point on the detector interferes with itself at the source. There is no need for the waves from separate points on the detector to interfere with one another. The processes connecting the source with different detection points are, for single particles, entirely independent.

The two elementary waves that actually stimulate the two photons in this example do not have parallel polarization for a general angle $\theta$. This might appear to contradict the finding, using the present theory, that the two photons are emitted with the same polarization. However, if the polarizers are parallel, one will, in the elementary waves theory, always see both photons or neither photon. The probability that one photon will be stimulated by the wave along the polarizer axis, and hence be observed, while the other photon will be stimulated by the perpendicular wave from the other side and then not be observed is $\cos ^{2} 90^{\circ}$, or zero. When the two polarizers are oblique, the waves stimulating the emitted photons are oblique; but this does not contradict what is actually observed experimentally. Whatever angle one uses, the probability of coincidence is exactly that predicted by current quantum theory.

Furthermore, as explained in the previous section, there is no need in this theory to assign any "spin" to the particle itself. All the spin behavior is captured by the waves, which, again, is exactly what the mathematics of current quantum mechanics says. The waves act as current theory describes, and the particle then "blindly" follows. Spin, thereby, acquires a simple, pictorial explanation.

It is necessary, however, to explain delayed-choice situations and, in particular, the experiment in which one polarizer, initially oblique to the other, is rotated back into alignment with the other polarizer after the photon pair is emitted. If the wave "spins" are actually oblique, for oblique angles between the polarizers, and we then rotate the polarizers into alignment with each other while the particles are in flight, does the theory still predict the correct answer? Indeed, does it predict the correct answer for delayedchoice situations in general?

Consider a photon in flight from the source toward its polarizer. The polarizer is rotated, destroying the original elementary waves and creating new ones. The new waves arrive at the photon while it is somewhere between the source and the polarizer. But a particle must always follow an existing wave - the wave by which it was generated; it is that wave that determines the dynamics. If that wave disappears, the photon must jump into "coherence" with one of the new waves; it cannot remain in its original state because the corresponding wave is gone.

(In general, I will describe a particle as being "coherent" with the wave that it is following. This is, of course, a generalization of the usual meaning of this term.)

The jump of a particle into a new state while out in space, not interacting with any other (local) particles, might appear strange and/or arbitrary at this point. But when the process by which a particle follows its wave is described in more detail (Secs. 8 and 9), it will become clear that this is necessary and fits directly into the overall theory. The only observable effect of the jump is 
the subsequent interaction with the newly orientated polarizer. But, as we will see in a moment, the theory being offered predicts exactly the same results of that interaction as the current theory (for single-delayed choice), which, of course, agrees with what is observed experimentally.

Furthermore, current quantum theory actually predicts exactly the same phenomenon, although it is not pictured as such. Consider a "wave-particle" in a definite state in a box. If the box is changed, the "wave-particle" immediately jumps into a superposition of the newly available states. In Sec. 12 it will be shown that the mathematics describing the jumping process in current quantum theory is identical to that describing the jump in the elementary waves theory.

And remember here, again, that the particle photon itself does not carry a spin. Only the waves carry the spin; the particle then acts accordingly. So there is no need to conserve any angular momentum of the particle photon when the "jump" process occurs. Conservation of angular momentum is required only for the waves.

The detailed physics of a "jump" is rather complex but follows the pattern of the theory established up to this point. The "jump" of a photon involves the annihilation of the initial photon and the creation of a new one. The annihilation of the initial photon is equivalent to the creation of an antiphoton, that is, another photon, moving in the opposite direction. But in the elementary waves theory all particles are created in response to waves. To be consistent, this would have to include the effective (anti-) photon involved in the jump. Because the (anti-) photon moves in the opposite direction, it is emitted, in effect, in response to a wave coming from the direction of the photon source, a wave, that is, which is moving along with the initial photon. (This is not the wave being followed by the initial photon, but rather merely a wave moving along with it.) The new photon, which continues on to the polarizer, is emitted in response to the new waves coming from the polarizer. In effect, a pair of photons is created in response to the waves coming from opposite directions.

But a similar process occurs upon the initial emission of the photon at the source. An electron in an atom scatters and emits the photon. The electron is a pointlike particle following a wave, as is the photon, so the emission occurs at a single vertex. (More on this in Sec. 12.) At that vertex the scattering electron looks to the photon exactly as if another (anti-) photon had been created. The scattering electron can emit a photon so it is the equivalent, electromagnetically, of an (anti-) photon. So what one has, in effect, is again the creation of a photon pair, with the (anti-) photon corresponding to the electron scattering.

But, again, as with all particles in this theory, the effective (anti) photon is emitted in response to a wave. Because the (anti-) photon is absorbed by the electron, that wave must come from the electron. (All charged particles "emit" photon waves, as described in Secs. 8 and 9.) This (anti-) photon wave captures the spin orientation of the emitting atom - of the scattering electron. It is the angle between the polarization of this (anti -) photon wave and the photon wave coming from the polarizer that gives one the $\cos ^{2} \theta$. (Again, I am describing here the emission only of the second photon, as if it were part of a two-step and not a single quantum process. The more accurate description will be given in Sec. 12.)
Furthermore, because the photon and the effective (anti-) photon move in opposite directions, the (anti-) photon wave from the electron moves in the same direction as the photon. That is, it travels with the photon. So it is this very same wave that is present when the photon "jumps" later on. Hence the "jump" occurs exactly as it would have occurred had the new waves from the rotated polarizer arrived at the photon source before the initial emission. The photon pair process at the jump is exactly the same - in response to exactly the same waves - as that which would have occurred at the source had there been no delay. The result is exactly as if no delay had occurred.

In general, all particles will be accompanied by the wave that affected the (effective or actual) antiparticle involved in their emission, for reasons to be explained in Sec. 12. So if one changes the wave being followed by that particle in a delayed manner, and a jump to a new state occurs, the same pair process takes place that would have taken place had the new waves arrived before the particle's initial creation. The result is exactly as if there had been no delay. (However, as will be shown in Sec. 7, for massive particles the waves do not travel at the same velocity as the particles. Hence a new class of delayed-choice experiments, in which the waves following along behind a particle are changed after its emission, might yield some interesting results.)

Notice, then, that it is not necessary in general for a wave to make the entire trip from detector to source in order to understand quantum processes. If the wave changes while the particle is in midflight, the particle jumps into exactly the state it would have been in had the change occurred before the particle's creation at the source. With this fact one can understand how the elementary waves theory explains dynamic, changing systems as well as the static systems treated in Sec. 3.

There is one circumstance, however, in which the predictions of the elementary waves theory differ from those of standard quantum mechanics: double-delayed-choice experiments, in which both polarizers are independently rotated after a photon pair is emitted. When this occurs, each photon jumps into a new state with a probability that depends on the original orientation of the opposite polarizer, not its new orientation. The respective antiphoton wave involved in each jump will reflect the initial wave from the polarizer on the opposite side and not the new wave that appears after the rotation. So one will no longer obtain the quantum mechanical $\cos ^{2} \theta$ form.

It might be thought, then, that the experiment of Aspect ${ }^{(5)}$ refutes the elementary waves theory. However, Aspect did not simply change each polarization once in a delayed manner. In his experiment each polarization was switched rapidly back and forth between two particular polarizations using an optical commutator. Furthermore, the distance between each commutator and the photon source was chosen as twice the distance $D$ that light can travel in the time that the commutator remained in one condition. ${ }^{(5)}$ As a photon travels from the source to the commutator, in the elementary waves theory, it will experience changes in its elementary wave due to the commutator switching. But a quick check shows that, because of the above factor of 2 in the distance, when the photon arrives at the commutator, the commutator will always be in the same condition that it was in when it transmitted the wave that stimulated the initial emission of that photon. So 
Lewis E. Little

even though the photon might have jumped back and forth between the two different wave sets as the commutator switched, it will end up in the same state at the commutator that it was in when it was emitted, and the commutator will be in the same state that it was in when the wave was transmitted. The net result will be exactly as if the commutator had never changed. The factor of 2 nullifies the effect that was to have been observed in the experiment.

In order to serve as a test of the elementary waves theory, the distance from commutator to source would have to be a half-integral multiple of the distance $D$. If the distance were, say, 2.5 times $D$, that is, if the separation between the two commutators were 5 times $D$, the experiment would be a valid test. I predict that if the experiment is repeated with the half-integral separations, it will not reproduce the present quantum predictions.

In all respects other than double-delayed-choice, this explanation of photon EPR exactly reproduces the predictions of quantum mechanics. Some of the mathematics is different, due to the fact that no "entangled" wave functions are required; otherwise, the mathematics of the waves is identical. The theory is local and deterministic; both the waves and the resulting particles follow local, deterministic laws. No nonlocal "collapse" of an entangled wave at the polarizers is involved. The key is the fact that the square occurs at the source, at which point the decaying system has information regarding the orientation of both polarizers.

There is some unpredictability, as opposed to indeterminism, in this theory, in that we do not know in advance which wave the source will respond to in emitting a particular particle photon. However, unlike the situation in the usual theory, here the unpredictability can be described as resulting from a random process following an ordinary probability distribution. All the wave states exist as real waves. The source then simply has a constant probability of responding to the intensity of each incident wave. The randomness thus reflects lack of knowledge of the value of some parameters in the source, rather than representing a fundamental indeterminism.

The "hidden variables" must, mathematically, come into play as part of the event at which the squaring of the wave is performed. In current theory this is at the detector. But in fact the hidden variables are in the source, not the detector.

The explanation of EPR experiments using particles other than photons, or of experiments involving parameters other than spin, exactly parallels the case for photons.

Whether or not the elementary waves theory is correct, this theory of EPR experiments clearly constitutes a counterexample to the conclusions usually drawn from Bell's analysis. ${ }^{(1)}$ It is indeed possible to explain EPR experiments with a local, deterministic theory. Bell's theorem, coupled with the experiments confirming the associated quantum mechanical predictions, does not "refute reality," as is so frequently claimed.

\section{THE UNCERTAINTY PRINCIPLE}

The elementary waves theory explains the appearance of "wave-packet" phenomena and hence gives a physical explanation for the uncertainty principle. Perhaps the best way to picture this is with the experiment of Kaiser et al., ${ }^{(12)}$ illustrated in Fig. 3. This experiment employs a Werner-type ${ }^{(13)}$ crystal neutron interferometer, with a bismuth (Bi) sample in one arm to delay the beam. An analyzer crystal is also placed in front of one of the detectors in an exit beam to select a narrow wave band from the wider bandwidth that is otherwise accommodated by the interferometer. If the $\mathrm{Bi}$ sample is made large enough, interference, in the absence of an analyzer crystal, disappears. The explanation given by the current theory is that the wave packet is not long enough to maintain the coherence: the coherence length is too short. Interference disappears, because, with the delay due to the bismuth, the packets traveling on the two arms of the analyzer no longer overlap at the final crystal plate.

However, with the analyzer crystal in the exit beam one can narrow the observed bandwidth further. And, as if by magic, the interference returns, even with the larger $\mathrm{Bi}$ sample in place; varying the width of the Bi sample now makes the beam reflected by the analyzer crystal come on and off. This is interpreted by Kaiser as implying that the subsequent action - after traversing the interferometer - of narrowing the bandwidth affects the prior bandwidth of the wave packet and hence its coherence length, one of many examples of reverse-temporal causality in current quantum mechanics, which, of course, makes no sense.

In the Kaiser experiment what is actually happening is that each detector is emitting elementary waves back through the system at each frequency in the full bandwidth that the interferometer will accommodate. With no Bi sample inserted, the interference, now at the first plate, of the waves is such that all frequencies within the bandwidth exhibit the same interference. Thus all the waves from a particular detector will go one way - for a perfectly aligned interferometer - as they leave the analyzer, either toward the particle source or along the other direction. What we have is exactly the usual quantum interferometer, but with the waves moving in reverse. Thus all particles from the source will go one way in the end, toward the detector that emitted the waves that reached the neutron source and that the neutrons are thus following. (What I have just described, then, is how the elementary waves theory explains interferometers. And as with all systems, the particles need take only one path; the waves take both.) As one inserts a little $\mathrm{Bi}$, all frequencies still exhibit the same interference. But with enough $\mathrm{Bi}$, different parts of the bandwidth begin to exhibit different interference. The delay due to the bismuth creates a different phase shift depending on the wavelength of the wave. Some parts thus go one way and some the other, and the interference is washed out. Waves from both detectors arrive at the source, and so particles then arrive at both detectors.

However, if one inserts the analyzer crystal to single out a narrow band of the frequencies, the interference is found to still be there. That is, all the elementary waves emitted by the detector behind the analyzer crystal and then selected by the crystal will interfere in a common manner when they reach the first plate of the interferometer. The bandwidth selected by the analyzer crystal is now too narrow for the bismuth to produce phase shifts that differ enough from one another to produce a significant effect. Hence one will either see particles or not, depending on the particular frequency band that has been selected by the analyzer 
crystal.

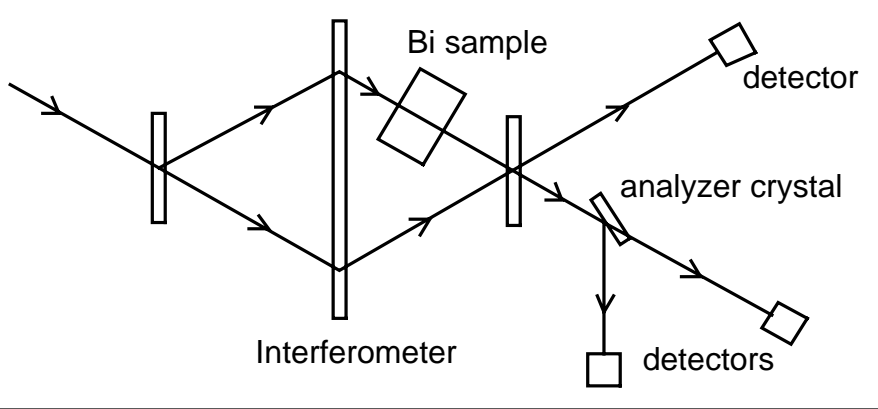

Figure 3. Experiment of Kaiser et al.

One can imagine performing the Kaiser experiment with a large, fixed Bi sample, but with a variable analyzer crystal. As one swept across the wideband-width with the narrow-range analyzer, the observed particle beam leaving the analyzer would go on and off. But these peaks and valleys are mixed together in the exit beams when the analyzer is removed, which is why one sees no apparent interference.

Quantitatively the relationship between $\Delta x$ and $\Delta p$ is the same as in current theory. As an approximation, describe the bandwidth accepted by the interferometer as having a width $\Delta \lambda$ centered on wavelength $\lambda$, with all frequencies within this width having equal amplitude. Then interference will be completely wiped out when the bismuth causes the waves at one end of the bandwidth to shift by $2 \pi$ relative to those at the opposite end. For each wavelength $\lambda$ by which the wave is delayed by the bismuth, the shift of the two extreme waves relative to one another will be $\Delta \lambda$. So to get the full shift of $\lambda$ one needs a number $n$ of wavelengths given by

$$
n=\lambda \Delta \lambda
$$

The total distance by which the wave is shifted by the bismuth is then this times $\lambda$, or

$$
\Delta x=\lambda^{2} / \Delta \lambda
$$

This gives the coherence length of the alleged wave packet.

But the momentum is given by

$$
p=h / \lambda
$$

so the uncertainty in $p$ is (by simple differentiation)

$$
\Delta p=-h\left(\Delta \lambda \lambda^{2}\right)
$$

Hence, using absolute values for the uncertainties,

$$
\Delta x \Delta p=h
$$

So one sees how the wider bandwidth gives a shorter apparent coherence length and hence the appearance of a shorter wave packet, and vice versa. The accuracy of one's "knowledge" of the frequency/momentum is thus inversely proportional to the accuracy of one's "knowledge" of the position, which is the uncertainty principle.

But this entire "uncertainty principle" way of looking at things is necessary only in a theory that holds that the particle is the wave. With the elementary wave picture it is clear that there is no actual uncertainty at all. Indeed, there are no wave packets at all. Every individual wave frequency acts independently from all others, and every particle follows its own individual wave.

Remember that in present quantum theory one can treat a general scattering process either by individual frequency waves or by wave packets. The results are identical. There is no need to have any "glue" to stick the various frequencies together in a packet. All frequencies act with complete independence.

What forces one to assign a fundamental uncertainty to particles in current theory is the forward motion of the waves. By assuming that the wave goes from source to detector and that the wave is the particle, one is forced to conclude that the particle exists in multiple states simultaneously in order to explain phenomena involving "widths." But with the correct direction of motion one can understand the phenomena of "widths" without the need for any uncertainty in any parameter - without the need to assume that the particle itself was in all the states in the width simultaneously. Only the waves were in all the states, not the particle. And the existence of waves in all the states simply means that there was more than one wave involved, not that a single wave was in multiple states. Each wave is in one state at one time, as is the particle.

The exact value of the particle momentum is unpredictable. We don't know which wave will lead to the emission of a particle at which time and hence do not know in advance the value of the parameters describing a particular particle. But this is now due solely to ignorance of the value of parameters in the emitting system and not to any fundamental uncertainty.

There must indeed be such parameters in the emitting system to explain why it reacts to one wave rather than another, as indicated earlier. And these parameters are additional to those in standard quantum mechanics. They thus do constitute "hidden variables" in the usual sense. But it is clear now that they create no conceptual difficulties.

All "unpredictability," as distinguished from "uncertainty" in the usual quantum mechanical sense, is now explained as resulting from lack of knowledge of the values of parameters in the particle source. Hence there is no need to conclude that there is any lack of strict determinism. The "uncertainty principle" is thereby explained.

Further investigation will be necessary to determine the nature of the parameters involved. However, the fact that some such parameters can in principle account for the unpredictability of quantum phenomena has been demonstrated.

As an aside I must say that the notion of "hidden" variables of any kind is a misnomer. If a variable really were hidden, this would imply that it had no observable consequences, in which case one would never know of its existence; it would play no role in any theory. Indeed, a proper empiricism dictates that any such "variable" would be entirely meaningless. If a variable has any observable consequence, then, by that very fact it is not hidden. 
The "hidden variables" in the source above clearly do have observable consequences: the emission of one particle rather than another, and at a particular time. They are therefore clearly not hidden. A more correct designation would be "more indirectly observed variable." (All variables are observed "indirectly." The "hidden" variables indicated above are simply observed more indirectly.)

"Tunneling," usually thought of as an expression of the uncertainty principle, is simply explained by the elementary waves picture. The dynamics of particle motion is determined by the waves; and the waves obey the same laws as in current theory. In current theory, the waves "tunnel." So the elementary waves also tunnel, and the particles follow those waves. No uncertainty in the particle state is involved. This picture of tunneling will become clearer after the details of the process by which a particle follows its wave are presented in Secs. 8 and 9.

\section{SPECIAL RELATIVITY}

The elementary waves theory provides a simple, physical explanation for the fact that light travels at the same velocity $c$ relative to all observers and thus serves to explain the Lorentz transformation.

According to the theory, all particles obey a dynamics in which they follow a wave coming from the "detector." This is true of particle photons also, as seen in Sec. 4. Whenever we see a photon, our eye becomes the "detector." What we see is the particle photon, not the wave. It is the particle that imparts any energy or momentum to the retina, thus producing a visual effect. (Even though the momentum "resides" in the wave, a wave cannot impart momentum to a particle in the absence of another particle. Only a scattering with another particle can change a particle's momentum.) The same is true for any other object or "detector" that absorbs a particle photon. But if the dynamics of the particle photon is determined by a wave that comes from the observer, then it is the observer's frame that determines the velocity. The constancy of $c$ relative to the observer is thereby explained.

The elementary waves are not actually emitted by the observer, as indicated earlier. The observer merely rearranges the organization of the passing wave. It must be assumed, then, that the organization is imposed in such a manner that it reflects the frame of reference of the "emitting" particle. The particle photon that responds to that organization will then travel at velocity $c$ relative to the "emitting" particle. I will discuss this further at the end of the next section. For the moment, simply imagine that the waves actually are emitted by the observer, with the observer's frame thereby determining the dynamics. I will show that the actual situation is equivalent to this.

This explanation of the constancy of $c$, as will be shown below, does not require that a single wave travel the entire distance from an observer to the source of any photon seen by that observer, a proposition that would clearly be absurd for, among other things, intergalactic light. This need be true only for light observed locally, that is, for those distances at which our basic, directly perceivable units of length and time are established. The behavior of particle photons over long distances will be shown to be exactly the same as if a single wave made the entire trip.
Light, then, does not simply move from object to observer or from observer to object; it does both. Nor is it simply a wave or simply a particle. It consists of a wave from observer to object and a particle from object to observer. However, the fact that a wave travels from observer to object does not make this an "extramissive",(14) light theory, one in which light travels from observer to object. The light that is observed is the particle photons, which travel ("intromissively") from object to observer.

"Relativistic" phenomena can thus be understood without the requirement that space be a physical object of some kind that stretches and shrinks as we change frames of reference. What changes when one changes frames is only the light used to observe objects.

However, the fact that space does not change does not mean that one can dispense with Lorentz transformations and use simply Galilean transformations along with the change in the light. To see why Lorentz transformations are still necessary and why this does not conflict with the claim that space is unchanging, consider the following example.

Imagine for a moment that space-time were Galilean, and consider the experiment pictured in Fig. 4. Two lamps in the same frame of reference flash at the same instant as observed in that frame. An observer at the midpoint, and also in the same frame, will observe the light from both lamps at the same instant. The light moves at velocity $c$ relative to the observer.

A second observer is in a spaceship moving rapidly, with velocity $v$, in the direction from one lamp to the other. The timing of the ship's motion is such that the light from the lamp behind the spaceship arrives at the ship just as it passes the first observer. But that light is moving with velocity $c$ relative to the spaceship (the photons observed by the spaceship are following waves from the spaceship) and thus at velocity $c+v$ relative to the first observer - this, again, in our imagined Galilean universe. Light from the second lamp, similarly, moves at velocity $c-v$ relative to the first observer. Clearly, then, the light from the second lamp will not arrive at the spaceship at the same time as that from the first lamp; the light travels equal distances but at different velocities. But to the spaceship both light signals move with velocity $c$, and the distance traveled is the same (or would be if both signals reached the spaceship at the midpoint). So if the lamps fire simultaneously as viewed by the spaceship, the two flashes would be observed simultaneously.

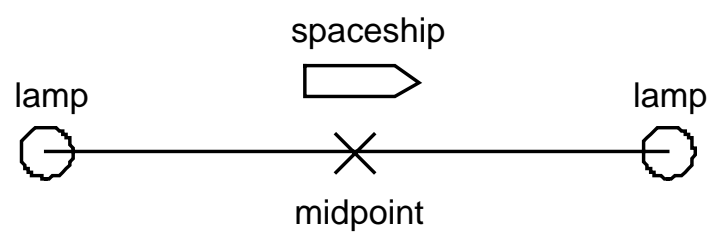

Figure 4. Illustration of "relativity" of simultaneity.

Because they are not, we must conclude that, to the spaceship, the flashes do not occur simultaneously, even though they are simultaneous to the first observer.

It is not simply the case that the lights appear to flash at different times. Even with a correction for the time of flight of the 
photons, the actual flashes of the lamps occur at different times as viewed from the spaceship.

We are thus forced to conclude that simultaneity is relative, even within this initially Galilean framework. But if simultaneity is relative, then so is length, as this is usually defined. If an object is moving, by its length we mean the distance, in the observer's frame, between the positions of the two ends of the moving object observed simultaneously. So given the relativity of simultaneity, we see that length will be relative also.

Indeed, given the constancy of $c$ - regardless of the physical reason for it - one can deduce the full Lorentz transformation. (So in the example of Fig. 4 both the simultaneity and the distance between the lamps will be different for the two observers exactly as in current theory.) The steps are directly parallel to current standard derivations. I will refer to two of them briefly in order to identify a few points of difference.

In one standard textbook derivation ${ }^{(15)}$ a flash of light is observed from all directions in two frames of reference, one moving relative to the other. Coordinate systems are defined in the two frames so that the two origins coincide with one another and with the light source at the moment of the flash. In both frames the light is observed to travel out from the origin in a spherical pattern, due to the constancy of the velocity of light relative to all observers. The Lorentz transformation is then derived as the transformation necessary to produce the light seen by observers in one frame from that seen in the other.

But according to the elementary waves theory, exactly these two spherical pulses are what would be seen by observers in the two frames. Imagine an array of observers in each frame, placed around the origin, but interspersed so they do not block one another. The light seen by each observer will move with velocity $c$ relative to that observer, because it is that observer's own elementary waves that will determine the velocity of the light he sees. The light will thus be seen by both arrays of observers as moving in a spherical pattern with velocity $c$. So the light seen by one array of observers is exactly what one would obtain by applying a Lorentz transformation to the light seen by the other array. The elementary waves theory thus predicts exactly the relationship captured by the Lorentz transformation.

In the standard derivation it is assumed that the light seen by both observers is physically the same light - the same photons. Space and time are then distorted in order to account for the fact that both observers see a spherical pulse. In the elementary waves theory observers in both frames still see a spherical pulse. But this is because the light is different, not because of a deformation of space-time. The two observers in two different frames do not see the same photons (this, again, for local observations where our units of space and time are established).

In this derivation it might appear as if what we have with the elementary waves is simply Galilean space with a change to the light. So it would be instructive for the reader to follow through another standard textbook derivation of the transformation, namely, that of Panofsky and Phillips. ${ }^{(16)}$ Every aspect of that derivation remains the same except for one change. The transformation for a time interval is derived by considering light that travels from a source to a mirror where it reflects and then returns to the source, this as observed first in the source's frame and then in a frame moving in a direction perpendicular to the light's direction of propagation. In this derivation the mirror used when the light is observed from the moving frame must be fixed in that moving frame, not in the frame of the light source. The light must consist entirely of light as it would be observed in the moving frame; so the mirror must be in that frame in order to emit the corresponding elementary waves. However, given that we obtain the Lorentz transformation anyway, the result is the same either way; the light arrives at the moving observer at the same instant regardless of which mirror is used.

It is clear in this latter derivation that lengths do in fact change when one changes frames. So, even though the space itself does not change, one nonetheless must use a Lorentz transformation to relate what is observed in one frame to what is observed in another. If this seems to be a contradiction, remember that a coordinate system is not the same thing as space. A coordinate system is a real object or imagined real object in space. An axis is the equivalent of a real ruler. A length measurement, as with all measurements, is not a measurement against some absolute standard, whatever that might mean. It is rather a comparison between two extended objects, one of which is taken as a unit. When one measures objects by comparison with a coordinate system, one is similarly comparing two objects. But if objects appear differently when moving, due to the change in the light used to observe them, the same will be true of coordinate systems. The coordinate system used in one frame, if viewed from another moving frame, will not look the same as the coordinates that one would use in that moving frame.

Given the fact of Lorentz transformations, all the consequences of that transformation occur in the elementary waves theory exactly as in current theory. Moving objects appear shorter, time intervals in moving systems appear dilated, etc. However, none of these apparent changes require any change to the objects themselves. Only their appearance changes, due to the change to the light.

What we call the length of an object when viewed from a moving frame - the distance between the end points observed simultaneously — is physically not the same thing as the length in the rest (or any other relatively moving) frame. Because simultaneity is relative, if one wants to get the same physical quantity in the moving frame, one would have to use nonsimultaneous times. It is only if one mistakenly holds that the "length" in the moving frame is the same physical quantity as the length in the rest frame that one will think that a moving object has shrunk. A moving object does not shrink.

The invariant quantity, the actual, objective nature of the object observed, is exactly what current theory says: the invariant interval. That interval is not simply mathematically equal in all frames; it is physically the same thing. The interval appears to change physically, because the "mix" of space and time is different in different frames. But this is entirely due to the change in the light, not to a change in the nature of the interval. All observers see the same reality.

The very definition of length, for a moving object, involves time - simultaneity - as indicated above. And the very definition of time involves length. Time is the measure of motion. It is by comparing motions - over distances, or lengths - that 
we arrive at a concept of time. So it should come as no surprise that the two concepts end up being "mixed" together as in the Lorentz transformation. It is specifically the motion of two observers relative to one another that affects the means of observation. But motion means length over time. The surprise, then, would be if there were no "mixing." But the fact that lengths and times change under transformation does not mean that an object itself changes.

What we have traditionally called length and time are inextricably tied up with the nature of our (principal) means of observing objects: light. The nature of light as a particle following a wave from the observer dictates that simultaneity is relative. This in turn forces us to use Lorentz transformations, even in a space that is unchanging. Lengths and times thus become "mixed."

It is thus clear that there is no contradiction involved in the fact that two observers in relative motion each see objects as being shorter in the other observer's frame. The apparent "shrinking" effect is reciprocal. Similarly for time dilation. The "twin paradox" in its various forms is thereby resolved.

The elementary waves theory of "relativistic" phenomena is an objective theory of those phenomena. Reality is the same for all observers. It is not the case that "everything is relative."

What, after all, do we mean when we speak of what exists objectively, independent of our means of observation? It means that, whatever means of observation we use, we subtract its effect from what we see in order to determine what was due to the object itself aside from the method of observation. Ordinarily, we think that for visual observations of position this can be accomplished simply by taking into account the velocity of light. We notice when we see the light pulse, we take into account the time it took the light to travel, and we then determine where the actual emission occurred and when. But this ordinary means of removing the effect of the light is actually premised on a Galilean view of things. This does not actually remove all the effects when we observe a moving object. To completely remove the effects of the light requires - exactly as we just showed above - that we do a Lorentz transformation. The use of different light does not just mean that the velocity changes. Also apparent distances change, time intervals change, simultaneity changes, etc.

The difference between what two observers see, as the result of using different light, is exactly described by a Lorentz transformation. So that, exactly, is what we must perform to remove the effect of the use of different light, thus insuring that what remains is physically the same for the two observers. Because what remains is physically the same, it must act the same. Hence all physical laws must be "covariant." Covariance, then, simply means that one has removed the effects of the means of observation.

Space, after all, is nothing. Space is merely the place where real objects can be located. What is real are the objects, not the space. We arrive at our concept of space by abstraction from real objects. So space as such, aside from the objects located in that space, can be neither Galilean nor Lorentzian, nor have any other special properties. Nothingness cannot have properties. If we assign any properties to space, what we mean is that these are properties that would be possessed by any object that might be located in space. If all objects transform in a Lorentzian manner, one might then say that space-time is Lorentzian. But this must not be understood as implying any modifications to the space as such. Nothingness cannot be modified.

It will be demonstrated in Sec. 14 that general relativity can also be understood without attributing "curvature" or other properties to space as such.

The elementary waves theory is "automatically" relativistic — it is already relativistic as it stands. It is not necessary to add relativity to a nonrelativistic theory. Had relativistic phenomena not yet been discovered, the elementary waves theory would have predicted them. I offer this as the single most significant piece of evidence supporting the theory. The same theory that explains quantum phenomena, immediately - with no further assumptions — predicts and explains special relativity.

Quantum mechanics and relativity are, indeed, one and the same theory. This explains the "intimacy" between quantum mechanics and relativity that was discovered when quantum mechanics was made relativistic.

With the insight that it is something moving from the observer that produces relativistic effects, namely, the elementary waves, we see what is not obvious from the current presentations of relativity theory: the theory is - in those current formulations a thoroughly nonlocal theory. If observed from a moving frame, an object is shorter. It does not just look shorter; it actually is shorter. So if one gets up and moves across the room, the fact of one's motion causes every (initially stationary) object in the universe, to its farthest reaches, immediately to shrink. It would be hard to imagine a more nonlocal theory. (Of course, objects stay the same for an observer who remains seated; but this merely illustrates the obvious contradiction involved in any theory in which the nature of things is "relative" to the observer.)

Indeed, turning this argument around, the fact of relativistic phenomena is the single largest piece of evidence that something must be traveling from the observer/detector to the particle photons. Without this there is no local means of understanding how objects change - or appear to change - when one moves. This, then, must be added to the list of evidences of reverse motion in Sec. 2.

Objects do indeed appear to change when one moves. But facts are facts; facts do not change because one looks at them differently. So one knows for certain that it is the means of observation that changes when one moves, not the objects observed. But motion of the observer can affect the means of observation only if the means involves something traveling from the observer.

Therefore, rather than starting with quantum phenomena and applying the "prequantal" philosophy, one might just as well have started with relativistic phenomena and applied the same philosophy, a philosophy that one might then call also "prerelativistic." From this one would deduce the fact of the reverse motion of the waves; and then from that fact one would explain quantum mechanics. Relativistic phenomena alone provide a sufficient basis to deduce the elementary waves theory, at least for photons, provided one maintains the view that facts are facts. 


\section{7. "RELATIVISTIC" TRANSFORMATION OF THE WAVES}

Consider a particle following its elementary wave as in Fig. 5. The particle moves to the right, the wave to the left. The energymomentum of the particle is related to the wavelength and frequency of the wave in the usual manner.

Suppose we transform to a system moving to the left as shown. The particle will be moving faster in the new system and hence should have a larger energy-momentum. In order for the theory to be invariant, the wave must similarly transform to a wave of higher energy-momentum. Otherwise, a particle of one energymomentum would appear in the transformed system to be following a wave of the wrong wavelength. But this can only happen if the wave fronts are moving to the right, with the particle. We thus seem to have a contradiction: the wave fronts move to the right, but the wave moves to the left.

However, the wave is present at all times. The effect of the particle or particles "emitting" the wave is not to generate the wave - there is no oscillation of the source as in usual wave emission - but rather is to establish coherence in the already existing wave. Furthermore, the phase velocity of a particle wave is given by $c^{2} / v$, which is an unphysical velocity anyway. So it cannot be the case that the wave fronts carry the wave signal, as would be the case for a wave in a medium. (This is one reason why the waves must be "elementary" and not waves in a medium.) The coherence signal and the wave fronts must propagate independently.

Remember that the usual resolution of the problem of unphysical phase velocities - using group velocity for a packet - is no longer applicable in this theory. Here there are no packets, as demonstrated in Sec. 5.

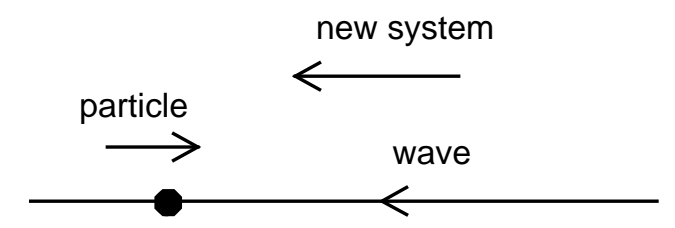

Figure 5. Transformation of particle following wave.

But if the wave fronts do not carry the signal, then there is noreason why the wave fronts might not move in either direction relative to the signal propagation.

As an analogy, consider an infinitely flexible, stretched string that can move in either direction along its length, but which at the same time can oscillate in a direction perpendicular to the string, this independently at every point along the string. With appropriate coordination of the oscillations at each point, one can make the string look like a wave at any instant of time, and the wave fronts can be made to travel in either direction at any velocity, including velocities greater than $c$. But such wave fronts would not carry any information or signal, due to the infinite flexibility, due, that is, to the causal independence of the motion of each point of the string. Any signal is carried by the string itself as it moves along its length.
The elementary wave objects are like the moving string. They are not waves in a medium. The elementary waves are the medium: they are the "material" filling otherwise empty space. They move with velocity $c$ (as will be shown in a moment), and the phase velocity can be in either direction relative to this actual velocity. There is no propagation of a signal through the wave. Rather, the wave object itself moves with velocity $c$, and thereby carries whatever coherence has been implanted on it. The coherence velocity, the velocity with which the coherence implanted on the wave travels, is the actual velocity $c$ of the wave object.

As strange as the notion of phase velocities being in reverse might seem, we will see in Sec. 9 that this is essential to the understanding of Feynman diagrams.

I will call an elementary wave with phase velocity in the opposite direction from the velocity of the wave object a "positive phase velocity wave," or a "positive phase wave" for short. If the phase velocity is in the same direction as the wave object, it is a "negative phase wave." The wave in Fig. 5 is thus a positive phase velocity wave. Even though the wave object moves to the left, the phase velocity is to the right, with the particle.

Negative phase waves must not be confused with "negative frequency waves." The latter appear in the elementary waves theory just as in current theory. The negative frequency waves are in fact positive frequency antiparticle waves. Both particle and antiparticle waves can have positive or negative phase velocity.

By having the phase velocity in the direction of motion of the particle, we achieve invariance for that velocity; the wave and the particle transform correctly together. However, the overall picture is still not invariant. The phase velocity of the wave will transform correctly, but we still must transform the wave or "coherence velocity." That velocity is opposed to the motion of the particle; this fact is the essence of the entire elementary waves theory. There is only one way that the overall picture can be invariant: if the coherence velocity of the wave is $c$, the velocity of light. Then it is $c$ in all frames, and the overall picture is invariant.

To summarize, a plane elementary wave is like a flux of material with velocity $c$, along any flux line of which has been implanted a wave (which varies with time in a manner that will become clearer in a moment). For a single coherent plane wave the wave on every flux line looks the same, has the same phase. The wave fronts will appear to move with a phase velocity that is greater than $c$, either positive or negative. But, as with the string above, there is no actual propagation of the wave along the material. Nothing actually moves with a velocity greater than $c$.

If a detector continually emits such a wave, with positive phase, then once the wave has been set up between the detector and some particle source, the resulting wave object along any line between the two looks exactly like the usual forward-moving quantum wave along that same line. (This will be demonstrated more fully in the next section.) So, as indicated in Sec. 2, the sign of the exponential describing the wave actually needn't be reversed. The wave looks mathematically identical to current quantum waves even though its propagation is reversed.

I am reluctant, however, to refer to a wave "material," as if it were something aside from the waves. There is no evidence of such a material. Indeed, if the wave objects are genuinely 
elementary, then it is meaningless to refer to a (more elementary?) material out of which they are composed. One can only say for sure that the wave objects exist.

Elementary waves are waves only in the sense that they add and subtract as waves when they are mutually coherent. That is, they so add and subtract insofar as they act to stimulate the emission of any particles. No actual cancellation of waves occurs; all "pieces" of every wave are present at all times. It is only the effects of a wave that cancel when its "pieces" are mutually coherent (and out of phase). This is unlike current wave theory, but is actually necessary in a theory where the waves are real things. The real waves do not go out of existence when they interfere; only their effects disappear.

What we end up concluding, then, is that space is filled with waves of all frequencies and wavelengths, all of which move with velocity of (coherence) propagation equal to $c$. Particle photons follow the photon waves (in reverse) with velocity $c$. Given the Lorentzian nature of space-time, where this is to be understood in the sense indicated at the end of the previous section, this "medium" of waves appears the same in all frames of reference. A given wave will appear to have a different frequency in another frame; but another wave will take its place in the new frame. What we have, then, is an "aether" of sorts, but one that is Lorentzian in nature. Rather than having a material medium through which the waves propagate, with the medium thereby fixing a preferred frame of reference, the waves themselves are the medium. They move with velocity $c$ in all frames, so there is no preferred frame.

The existence of a medium through which the waves propagate would clearly contradict this entire picture. One must view the waves as constituting the medium and thus as being elementary. The fact that space-time is observed to be Lorentzian, that is, that objects in space-time transform in a Lorentzian manner, is the primary evidence that the waves are indeed elementary.

Given that the phase velocities are not signal velocities, it is necessary to show that the wave, viewed as a geometrical object spread out over space, will transform correctly. That is, applying a Lorentz transformation to the space-time coordinates of all parts of the object should produce a new object with the appropriate wavelength - the wavelength corresponding to the appropriate momentum particle. While this follows also in current theory, it is not generally spelled out in treatments of this subject.

Consider as an example the wave corresponding to a stationary particle. Its wavelength is infinite. It oscillates with the same phase over all space with a frequency $m c^{2} / h$. Consider how this would appear to an observer moving in the $-x$ direction with velocity $v$. A particle at rest in the first frame will now move with velocity $v$ in the $+x$ direction. Because of its motion, clocks that were synchronous in the rest frame become asynchronous. This means that the phase of the wave motion will now appear to be different at different points in space; the oscillations now take the form of a traveling wave.

At a distance $L$ in front of the moving observer, as measured in the observer's frame, clocks will appear to be ahead by an amount $^{(16)}$

$$
\delta=\frac{L v}{c^{2}\left(1-v^{2} / c^{2}\right)^{1 / 2}} .
$$

Or, in the direction of motion of the particle, the clocks in front appear to be behind by that amount. The distance $L$ corresponds to one wavelength $\lambda$ of the wave when $\delta$ equals the period $h / m c^{2}$; so we conclude that

$$
\frac{h}{\lambda}=\frac{m v}{\left(1-v^{2} / c^{2}\right)^{1 / 2}} .
$$

But this is the correct expression relating the wavelength to the ("relativistic") momentum.

In general, changes to the phase velocity simply reflect changes to simultaneity resulting from a change in the relative velocity of the observer's frame. So in this manner we also see that the phase velocity cannot correspond to a signal of any kind. In the "rest frame" of a wave, that is, the rest frame of the particle that might follow that wave, there is a common phase at all points along the wave. The "traveling wave" time dependence that occurs in a moving frame results entirely from changes to simultaneity.

A similar analysis can be performed for transformations perpendicular to the motion of the wave. Again, the effect is one of a change to simultaneity (as well as a contraction of length in the direction of motion). The result shows that if a particle is moving perpendicular to the wave fronts in one frame of reference, it will move perpendicular to the wave fronts as viewed from any other frame of reference. (The wave fronts, however, will no longer be perpendicular to the direction of propagation of the wave.) In general, then, the picture of the wave objects as propagating in the direction opposite to the particle, but with the wave fronts moving with the particle with phase velocity $c^{2} / v$, and with the particle moving in a direction perpendicular to the wave fronts (by a mechanism to be explained in Sec. 9), that picture transforms correctly between frames. (Although the particles move perpendicular to the wave fronts, those wave fronts play no role in the particle's motion; only the wave along the line of motion of the particle has any effect on the particle.)

Returning to an earlier point: as a photon travels, the wave that it is following will frequently be disrupted due to motion of the wave source or to the intervention of other objects between the photon and the wave source. So the photon will have to "jump" waves, as described in Sec. 4. However, given that all elementary waves travel with velocity $c$, we see that the photon's velocity will not be affected by the jump. Its velocity when following the new wave will be the same as when following the old. And the direction of motion will also not change during a jump. (To change direction an additional particle would have to be involved.) So photons traveling over any distance will always travel with velocity $c$ in a straight line (aside from gravitational effects), which, of course, is what is observed. A single wave need make the full trip only for "local" light signals, this in order to explain the Lorentzian nature of space-time.

Furthermore, as demonstrated in Sec. 4, whenever a jump occurs, the state of the photon after the jump is exactly the same as 
it would have been had the new waves traveled the entire distance to the photon source before the emission. What one sees when a photon arrives is thus exactly what would have been seen had the wave made the entire trip. Only the state of motion of the observer at the instant the photon is observed will affect what is seen. This is genuinely an "intromissive" theory.

The final picture of the waves that we have arrived at might appear to contradict the initial physical explanation for the constancy of $c$. Every observer, it was argued, sees particle photons as moving with velocity $c$ because they follow a wave from that observer. But now we have concluded that all waves move with velocity $c$, as do all photons. So how is the wave from one observer any different from the wave from any other observer? And if they are not different, how does the original explanation work?

The problem here is the assumption that it is the velocity of the wave that determines the velocity of the photon following it. An essentially Galilean model of the situation is assumed, in which a wave will move with a fixed velocity relative to the source; so if the source is moving, the wave will move with a different velocity, as will the particle photon which then follows that wave. But clearly that kind of picture will not work here; all waves move with the same velocity.

What one is forced to conclude is that it is the "organization" imposed on a wave that determines the velocity of a particle following it. Somehow the organization reflects the frame of the particle that "emitted" the wave, and the particle following the wave then moves accordingly.

The constancy of $c$ requires that a photon's velocity be causally determined by the frame of the observer. Since the velocity of the waves is not unique to a particular frame, it cannot be the velocity that causes the frame dependence. It must be that the organization itself is frame-dependent.

This is connected with a point made at the beginning of the last section. The waves are not actually emitted by a particle, but rather are only rearranged as they pass by. So if the velocity of the wave were what determined the velocity of a particle photon following it, that particle velocity would not be $c$ relative to the wave "emitter," but rather would be $c$ relative to a frame determined by the wave aside from the "emitter." We would then have a Galilean and not a Lorentzian theory. But if the moving particle responds instead to the organization of the wave, and if that organization in turn reflects the frame of the wave "emitter," then the explanation for the constancy of $c$ still works. The "emitter" really does emit the "stuff" that determines the velocity of the particle: the organization.

The velocity of all photons will still be $c$ anyway, regardless of which wave a photon follows. But the fact that the velocity of a photon is always $c$ does not mean that the causal connection between the photon velocity and the organization of the wave disappears. Rather, it is only because of the existence of that causal relationship that space-time is Lorentzian and, therefore, that the velocity is always $c$ regardless of the wave source. From a causal point of view, the fact that waves from different sources are not distinguished by their velocity is, in effect, a coincidence.

So, for example, in the first derivation of the Lorentz transformation described in the previous section, the two sets of photons observed by the two arrays of observers actually both move with velocity $c$ relative to both sets of observers. But this does not mean that they are interchangeable. The photons are distinguished by the fact that they are following waves with a different organization. And it is only because of this fact that one obtains Lorentz transformations, which, by "coincidence," dictate that both sets of particle photons move with the same velocity $c$ relative to both arrays of observers, a fact that was certainly not apparent when one began the derivation. (Notice, then, that as matters turn out, even if an observer in one frame were to "jump in front of" an observer in the other frame, the photon that he would see would still be in the correct state to reflect his frame, given the above explanation for delayed choice.)

And there is no contradiction involved in saying that both sets of photons move with velocity $c$ relative to both arrays of observers, now that it is clear that velocity itself, involving length and time, will be affected by the means of observation. Velocity is length over time. But both length and time appear to change when we change frames. So what we call the velocity in one frame is physically not the same thing as velocity in another frame. It is only by considering the invariant interval that we would have the same physical quantity in both frames. But the interval for a signal moving with velocity $c$ is zero. So it will be zero in all frames.

A frame-dependent organization is, of course, not a new idea. The electromagnetic field of a charged particle is different depending on the velocity of the charge relative to the observer. By measuring the electric and magnetic fields at a point, both in direction and magnitude, one can determine the velocity of the charge emitting that field. The electromagnetic field, as will be demonstrated in Sec. 8, is actually itself simply an elementary wave, with particle photons then following the wave. The electromagnetic "organization" of the elementary waves reflects the emitter's frame.

The fact that the organization reflects the emitter's frame is actually essential to the ability of mutually coherent waves to distinguish between themselves and other, mutually incoherent waves. I will return to this point in Sec. 15.

\section{FEYNMAN DIAGRAMS I: QUALITATIVE INTERPRE- TATION}

Scattering experiments can be described using the picture for a general experiment given in Sec.3. Elementary waves are "emitted" by the detector, scatter off the target, and arrive at the source where they stimulate the emission of particles. The particles then follow the waves to the detector. The square of the wave amplitude at the source gives the cross section. The cross section will be exactly that of current theory because, by reciprocity, the wave scatters with the same matrix element.

Because the wave-scattering problem is in essence the same as that in current theory, one can perform the usual analysis and express the scattering in terms of matrix elements between plane waves. Furthermore, it is not necessary to the theory that the same coherent wave make the entire trip from detector to source. Indeed, for most particle scatterings outside the laboratory, the environment will be continually changing, and hence the wave being followed by any particle will be continually changing. For a 
Lewis E. Little

general scattering a particle will be traveling in the direction of a target while following one wave or another in free space. As it approaches the target it will jump into coherence with one of the waves scattering off the target. This wave will have originated from another plane wave incident on the target from some other direction. The particle follows that wave and will then be traveling in that other direction after the scattering. If a detector is located in that direction, eventually the particle will experience the waves coming from the detector, will jump into coherence with one of them, and then be detected.

In this picture the square of the amplitude, the square, that is, that determines the cross section, does not occur at a physical particle source, but rather takes place when the jump occurs into coherence with the wave from the target. The "source," in effect, is the oncoming particle beam.

Feynman diagrams, in this theory, actually picture accurately what is going on in the scattering, both when the waves scatter and when the particles subsequently scatter. The wave-scattering description is very similar to current theory, with the waves taking all the various (configuration space) Feynman paths. Just as for any other quantum system, there is no problem with the waves taking multiple paths; one simply has multiple waves. Each particle, when it scatters, will take only one path.

However, the elementary waves theory requires some essential changes to the interpretation of the diagrams. To illustrate these changes, consider electrons scattering off target muons in first order (Fig. 6). In the first place, the photon propagator waves that scatter the electron waves only become organized in the presence of a particle muon. In the absence of the muon, only disorganized photon waves exist, which produce various "renormalization" processes (see below), but not the wave processes corresponding to an electron-muon particle scattering. So at least the target muon particle must be present. This is unlike the usual theory in which the diagrams describe interacting wave fields. (Of course, the particle muon is itself following a muon wave.)

Furthermore, the electron wave scattering off the muon particle must occur in the absence of the particle electron. This is the whole idea of the elementary waves theory: the electron wave scatters toward the electron particle source, and the cross section is determined by the wave intensity at the source. So we must understand how Feynman diagrams describe the scattering of the electron waves from the particle muon, this in the absence of the particle electron. Then we must also understand how the very same diagrams describe the actual scattering of the particle electron when it arrives at the muon. I will describe the theory qualitatively at first, and then show the detailed correspondence to the mathematics of current theory.

The sequence of events is as follows: first the muon "emits" the photon propagator waves. The muon is following some muon elementary wave and might scatter into any of the other available states, that is, real elementary waves, around it. Even though it does not yet scatter, it emits the corresponding photon propagator wave. One can represent this as in Fig. 7.

This is a general property of particles: they "emit" all those waves corresponding to scattering processes in which they might participate, this in reaction to the incident waves corresponding to the other particles in that scattering process. The intensity of the emitted photon waves is given by an expression similar to that arising from the usual fermion "current," $e \bar{\psi} \gamma^{\mu} \psi$. Notice, however, that all potential photon propagators are being emitted, and not just the one corresponding to a particular particle scattering that will occur in the future. In present quantum theory the "current" means $e \bar{\psi} \gamma^{\mu} \psi$ for the specific final state relevant to the scattering. But one does not know which final state the muon will have until one knows to which propagator the electron has responded. But the electron cannot respond until the propagator has been emitted, and the propagator cannot be emitted until the muon scatters ... There is a clear lack of causality in this formulation. The problem disappears when the wave and the particle photon are separate things.

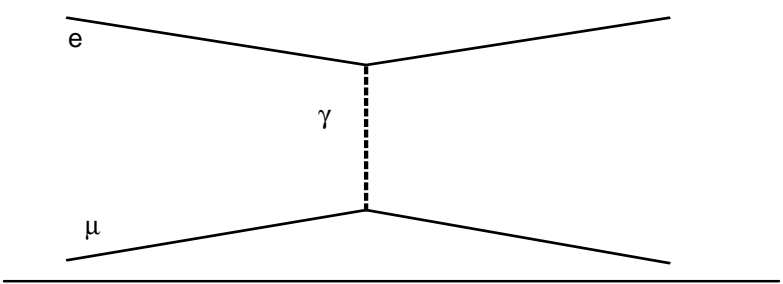

Figure 6. First-order electron-muon scattering.

In the figures the photon propagator is shown as a dashed line connecting particular points. This is simply to indicate that one is concerned with the effect of that propagator specifically at the vertex in question. Actually it propagates spherically in all directions as usual.

However, there is another important physical difference here from the usual theory, as indicated earlier. Elementary waves do not actually scatter from one direction to another. The wave flux in one direction continues in that direction indefinitely. At a vertex, or at a particle that interacts with the wave, the effect is one of rearranging the organization of the wave. The vertex, in effect, leaves a "shadow" on the passing wave flux - a line along which the coherence is reorganized. A spherical propagator is actually composed of waves that were incident on the originating point of that propagator from all directions, which waves are then reorganized at the vertex. A "shadow" is left on each passing wave. The sum of all the "shadows" looks like a wave propagating spherically out from the vertex, because the vertex imposes a common coherence on each shadow line. But actually the wave along any single line out from the vertex is independent of the waves along the lines in all other directions. The use of lines in the Feynman diagrams is thus not merely symbolic, but rather pictures the physics that is actually going on.

Notice here again, then, that there is no propagation of waves according to the usual wave dynamics, requiring a field equation, etc. Even spherical waves are entirely a product of simple, straight-line flux propagation.

When a particle or a vertex leaves a "shadow," it is not clear whether the wave in the shadow is fully or only partially organized. All that one can say is that the cross-sectional area of the particle or vertex, multiplied by the degree of organization 
imposed, is such that the "emitted" propagator is equal in intensity to that in current theory. Stated differently, the "charge" of a particle is proportional to this product. (However, the size of the particle or vertex must be small enough that it appears, from the point of view of other particles affected by the propagator, as if it were a point object.)

The photon propagator contains both positive and negative frequency waves as usual, that is, it contains particle waves

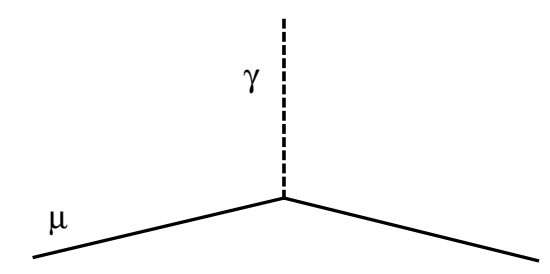

Figure 7. Emission of photon propagator.

moving in one direction and antiparticle waves in the other. (More on this below.) For photons the antiparticle is still a photon, of course. However, I will refer to the photons following the negative frequency waves as (anti-) photons in order that the analogous situation with mesons or other particles is clear. (If charged mesons were being exchanged, rather than photons, then clearly the positively charged meson would move in one direction and the negatively charged meson the other, this for the same scattering situation.)

Notice that the negative frequency waves are essential to the picture. Only the target muon is present when the electron waves scatter, so we must understand how the muon can emit waves corresponding to photons that will travel both to and from the muon. The positive frequency waves correspond to photons that will move toward the muon, the negative to photons that will move away. (This is the reverse of the usual definition, because here the wave moves in the reverse direction from the particle.)

Electron waves passing in the vicinity of the muon will be scattered by the photon propagator waves, again, just as in current theory. The amount of any electron wave that is scattered at a vertex, that is, the degree of mutual coherence established between the incident wave and any other wave at the vertex, is given by the same vertex function $e \bar{\psi} \gamma^{\mu} \psi$. The overall scattering will look very similar to the usual "current-current" form.

But there is a further important physical difference. The electron elementary wave objects propagate with velocity $c$, and not with the velocity $v$ of the particle electron or the phase velocity of the waves. So the timing of the vertex interactions of the wave is not the same as in the usual theory. However, the variable $t$ in the usual theory does not correspond to the actual motion of the particle or particles. That time corresponds to the phase motion of the waves that move along the various Feynman paths. It is the phase velocity that relates to the variable $t$ which occurs in the current mathematical expressions for the diagrams. Once the vertex interconnections of any diagram have been set up by the elementary wave objects - propagating between vertices with velocity $c$ - the resulting phase wave object will look identical to the wave object pictured in the usual theory by the same diagram.
Even the direction of motion of the wave fronts will look like the forward-moving waves of current theory, as explained above. A "snapshot" of the waves along the lines of a particular diagram with particular vertices will thus look identical to the object described by the same diagram in current theory. Integrating over all possible vertex locations will then yield the same result. The different timing of the vertex interactions changes nothing.

When two waves interact at a vertex, each wave "scatters" the other by the same amount; the usual vertex expression for scattering from state 1 to state 2 is simply the complex conjugate of the scattering from 2 to 1 . So the net amount of coherence does not change at a vertex. Whatever coherence is imposed on wave 2 by wave 1 , the initial coherence of 1 is reduced by the same amount as 2 imposes its coherence on 1 . There is "conservation of coherence."

The particular electron wave from the detector that is in question scatters from all points around the muon in the direction of the electron source (and in all other directions, of course). The electron, then, has some probability of being emitted in "coherence" with that particular wave, with a probability determined by the square of the coherent amplitude at the source.

When the electron arrives at the target muon, two things can happen, regardless of the particular location of the vertex at which the particle electron interacts. The combination of the photon waves from the muon, plus the electron wave from the detector with which the electron is coherent - can induce the electron to emit a particle photon and hence scatter. The particle photon then follows its wave to the muon and causes it to scatter. Or the (anti-) photon wave being emitted by the electron - just as the muon emitted its photon propagator waves - can stimulate the emission of a particle (anti-) photon from the muon, causing the muon to scatter, following which the particle (anti-) photon travels to the electron and causes it to scatter.

The (anti-) photon must follow a wave from the electron; otherwise, it will not arrive at the electron and cause it to scatter. However, that (anti-) photon wave is not present until the electron is present. This might make it appear as if the corresponding electron wave scattering cannot occur at the muon before the particle electron is present. However, again, this is the role of the negative frequency photon wave from the muon. The negative frequency photon wave from the muon produces the electron wave scattering that corresponds to the electron particle scattering caused by the (anti-) photon exchange.

Remember that it does not matter which path the electron takes anyway, just so it arrives at the detector by one means or another. Only the wave scattering is involved in determining the cross section (at the source). But, in fact, as just illustrated, the particle scattering can always mimic any wave scattering.

Notice, however, that when the photon is emitted by the muon, that occurs as the result of the interaction between an (anti-) photon wave from the electron, the muon wave being followed by the particle muon, and the muon wave into which the muon will scatter. The electron wave itself does not directly participate. However, the electron wave must somehow participate, because it is that wave that dictates the behavior of the electron. A particle photon unrelated to the electron wave would scatter the electron out of coherence with its wave. The only way in which the electron 
wave could participate would be if the (anti-) photon wave from the electron is produced by a potential scattering of the electron that will leave it coherent with its wave.

But this makes perfect sense in the above picture. The electron emits propagator waves corresponding to scattering processes in which it can participate. But once the electron has become coherent with a particular wave, it can only participate in scatterings dictated by that wave. The electron will not respond to incident electron waves with which it is not coherent and hence will not emit the corresponding (anti-) photon waves. So only particle (anti-)photons that leave the electron coherent with its wave will arrive at the electron, because only the corresponding (anti-) photon waves from the electron are present to stimulate the emission of those (anti-) photons from the muon.

If a wave is disorganized, a particle following that wave will, in effect, follow all pieces of the wave. It can then potentially scatter into the state of any incident particle wave. So it "emits" all the corresponding photon propagator waves. But once the particle has become coherent with a particular coherent, organized piece of the wave state, it can only respond to that coherent wave. If the coherent wave scatters at a vertex, then the particle, when it arrives at that vertex, can respond to the incident wave from the new direction, but not to any (mutually incoherent) particle waves from any other direction. So it emits only the corresponding propagator waves.

In a two-particle scattering, one particle is always the "leader." The leader is that particle which first responds to - "jumps" into coherence with - a coherent wave scattered by the other particle. From that point on the entire scattering process is dictated by the leader's wave, because any exchanged quantum, moving in either direction between the scattering particles, is dictated by that wave. The "follower" particle then scatters accordingly. In the above example the muon is the "follower," the electron the "leader." Momentum is always conserved in a scattering, of course, because any exchanged particle affects the momentum equally at both ends of its trip.

The above description extends to diagrams of all orders. The waves scatter by all possible Feynman diagrams simultaneously, and by all arrangements of vertices for any one (topologically distinct) diagram. The Feynman picture here is equivalent to a series of successive approximations, as in current theory. But the "heuristic" derivation of that picture, ${ }^{(17)}$ in which waves scatter at individual points and move on straight lines until scattering at another point, etc., describes correctly what is occurring physically.

Each infinitesimal "piece" of the wave scatters according to a particular diagram with a finite number of vertices. The evidence supporting this hypothesis will be indicated in Sec. 13. Each diagram is thus not, in fact, part of a successive approximation expansion. Each pictures a real process that is occurring. The diagrams add coherently because the resulting waves toward the electron source add coherently in stimulating the emission of the electron. (Here again we see the simple physical explanation of why one adds amplitudes.)

The particles also will always take a path with a finite number of vertices, separated by straight line segments. The particle path is "quantized," with discrete particle photons being emitted at vertices. It is the particle photon emission and absorption that causes the particle electron to scatter, not the waves alone. The waves dictate the dynamics of the process, but no particle scattering occurs in the absence of other particles.

An individual particle scatters according to one diagram, while the waves scatter according to all diagrams simultaneously. Do not be confused by the fact that I present the first-order particle scattering along with the first-order wave scattering. I am simply illustrating how the particle can "mimic" any wave diagram in reverse.

Notice that the particle can follow a path with a finite number of vertices only if the wave "pieces" scatter in a similar manner. Otherwise, at any one point the particle will experience a coherent wave coming from only one direction - resulting from the coherent sum of the waves in all Feynman diagrams - requiring the particle to follow a single, smooth path. But with scattering of the various pieces of the wave according to different Feynman paths with different vertices, the particle will experience coherent waves in many different directions at each location around the target. It will then choose one wave piece or another in a manner determined by parameters internal to the particle.

So the general lines of the theory are now clear. Waves corresponding to all possible free-particle states exist at all times. In the absence of particles the waves are "disorganized." Particles have the effect of imposing coherent organization on the waves. For any scattering process in which a particle might directly participate - with no intermediating particle - it "emits" the corresponding waves even when in a free state. That is, it imposes organization on those corresponding waves. Those organized waves then can cause the scattering of other waves in the vicinity. When a particle comes along following one of those scattered waves, it will scatter by emitting particles corresponding to the wave that produced the scattering of the wave it is following or by absorbing particles emitted in response to propagators that the particle itself emits, this in response to the stimulation of its own wave. The particle processes mimic exactly the wave processes in reverse.

As another example of a propagator that describes both particles and antiparticles, consider second-order electron-muon scattering, as in Fig. 8. The line representing the electron propagator consists of electron and positron waves. Particles can follow the diagram in a causal manner for both waves. For the electron propagator waves the electron simply follows the overall electron wave through the scattering, emitting or absorbing two photons in the process. If the propagator is a positron wave, then it must correspond to a positron moving in the reverse direction.

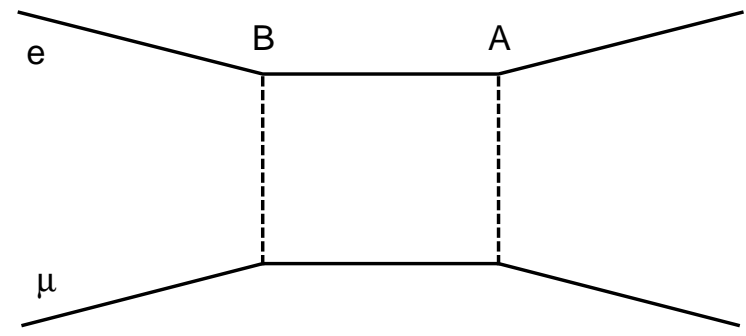

Figure 8. Second-order electron-muon scattering. 
So a pair must be created at point $\mathrm{A}$, with the positron traveling to $\mathrm{B}$ to annihilate the electron.

But, it might be asked, how does the pair "know" when to be created to produce the positron at $\mathrm{B}$ when the incident electron arrives?

The answer is that it is not the positron propagator wave that is responsible for producing the particle pair and carrying the particle positron. Rather, the incident electron itself emits positron waves. Again, a particle emits waves corresponding to any process in which it can participate. An electron can annihilate with a positron to form a photon, this at a single vertex (with either the photon or the positron or both off-mass-shell).

(Notice, by the way, that elementary waves must exist for all possible particle states, including off-mass-shell states. This simply means that waves exist corresponding to all possible particle masses. This is necessary, for example, to explain the quantum behavior of compound particles, such as molecules, which exhibit wavelike behavior as if a single unit.)

So the moving electron emits organized positron waves. As the electron approaches the muon, the positron waves from the electron can interact with the photon waves from the muon and the electron wave from the detector to generate a pair at A. The photon travels from the pair vertex to the muon, the electron follows its wave to the detector, and the positron follows the wave to the incident electron, where it annihilates, producing a photon that also follows its wave to the muon. So there is complete causality at every step, both for the particles and for the waves.

Do not be confused by the fact that I present the second-order particle process along with the second-order wave process. I am again simply illustrating how the particles can mimic any one of the wave diagrams in a causal manner.

And the probability that the particle follows one particular diagram or another is irrelevant to the cross section. The probability that the particle will follow a particular diagram might be entirely different from that of the corresponding wave diagram. Indeed, one cannot even compare the two because the wave diagram for specific vertices will interfere (at the particle source) with other arrangements of those vertices as well as with other (topologically distinct) diagrams.

Every aspect of this description of the microscopic details of particle interactions is strictly local. The waves interact only locally at vertices, with the interactions at each vertex depending only on the wave amplitudes at that vertex. A particle only interacts with the wave amplitude at the location of the particle. Particles only interact with other particles when at the same location.

No electromagnetic fields or potentials, aside from the elementary waves themselves, are required. One has only the real waves and the real particles. Notice, then, that it is the (four-dimensional) vector potential $A$ that is the real quantity, the real elementary wave. The electric and magnetic fields describe the resulting effects on particles and are thus derivatives of the vector potential.

There will be no problems with guage invariance in this theory, because one never need derive the vector potential from the electric and magnetic fields. The fields are rather the consequence of the potential, and there is always a one-to-one correspondence in that direction.

The ability to interpret Feynman diagrams pictorially in the above manner is made possible by the reverse motion of the waves. It is clear that with this theory the Feynman diagrams can picture what is going on physically without contradicting the uncertainty principle, or, in particular, the reasonings of Landau and Peierls. ${ }^{(18)}$ A completely causal, spatiotemporal picture of the scattering is obtained.

Because it is only the waves that take all paths, and not the particle, Weingard's arguments ${ }^{(19)}$ against the interpretation of internal propagators as corresponding to real particles are no longer applicable. Actually, the propagators correspond to both waves and particles.

\section{FEYNMAN DIAGRAMS II: QUANTITATIVE CORRESPONDENCE}

In the first-order electron-muon example of the previous section, the electron wave-scattering vertex might be located anywhere around the muon, and the particle electron might thus scatter at any location. To see how this happens, consider the propagator in more detail. Rather than the photon propagator, however, I will illustrate with the Klein-Gordon propagator in order to make more explicit the role of antiparticles and to simplify the mathematics. So imagine electron-muon scattering via Klein-Gordon particles. The propagator can be written as ${ }^{(20)}$

$$
\begin{aligned}
i \Delta_{F}\left(x^{\prime}-x\right) & =i \int \frac{d^{4} k}{(2 \pi)^{4}} \frac{1}{k^{2}-m^{2}+i \varepsilon} \exp \left[-i k\left(x^{\prime}-x\right)\right] \\
& =\int \frac{d^{3} k}{2 \omega_{k}(2 \pi)^{3}}\left\{\theta\left(t^{\prime}-t\right) \exp \left[-i k\left(x^{\prime}-x\right)\right]\right. \\
& \left.+\theta\left(t-t^{\prime}\right) \exp \left[i k\left(x^{\prime}-x\right)\right]\right\} .
\end{aligned}
$$

The latter form shows the division into positive and negative frequency waves. This propagator must be interpreted now as describing simply the propagation of the elementary waves and not as including any creation or annihilation of particles.

This is the propagator for a wave-particle in current theory to move from $x$ to $x^{\prime}$. Hence, because the elementary waves move in the opposite direction, it is the propagator for an elementary wave to move from $x^{\prime}$ to $x$. So in the example the muon is at $x^{\prime}$, the electron wave vertex at $x$.

The expression in the curly brackets, for a particular momentum $k$, describes two plane waves as a function of $x$. The positive frequency wave moves in the direction of $k$, the negative frequency wave in the opposite direction. (Remember again that the time variable refers only to the phase motion of the waves and not to the propagation of the actual wave object out from the muon.)

At any vertex $x$ observe that the propagator can produce a scattering for any $k$, including directions of $k$ that are not radial from the muon. If a Klein-Gordon $(\mathrm{KG})$ particle is to move 
radially from the muon to the particle electron and cause it to scatter, this at any vertex around the muon, then the KG particle must be capable of carrying momentum along directions other than its direction of motion. Indeed, if $k$ is the momentum transfer for a particular electron scattering, then when $x$ is in the $-k$ direction, the $\mathrm{KG}$ particle must carry a momentum in the opposite direction from its direction of motion.

It is here that the "negative phase" waves, discovered in Sec. 7, come into play. A particle can be emitted in response to a negative phase wave and can follow that wave in the usual reverse direction - reverse, that is, to the coherence velocity or actual velocity of the wave object. This means that it will now be moving in the opposite direction from the wave fronts. So it will carry a negative momentum. When absorbed by the electron it will "pull" rather than "push." Because of the negative momentum, the overall object still transforms correctly, even though the wave fronts now move in the opposite direction from the particle.

Remember again that "momentum," in the classical sense, is not actually carried by the particle anyway. What happens at the vertices is determined purely by the wave interactions. Because the wave is negative phase, the vertex interactions occur as if a particle carried a (classical) negative momentum.

Similarly, along any direction from the muon the $\mathrm{KG}$ particle can carry a momentum that is in the $k$ direction. The relationship between $k$ and the wavelength of the wave is given by

$$
\lambda=h / \mathbf{k}
$$

where is a unit vector from $x^{\prime}$ in the direction of $x$.

Consider a vertex located in the $+k$ direction from the muon, this for a scattering with momentum transfer $k$. The KG particle propagator wave is thus positive phase. At this vertex a passing electron would emit a positive momentum particle toward the muon. So the electron would recoil, and the KG particle, when absorbed by the muon, would cause the muon to recoil. It might be thought, then, that the negative frequency, antiparticle wave at that same vertex, which corresponds to the momentum $-k$, thus having negative phase, would have the wrong (negative) momentum. However, remember that the anti-KG particle is emitted by the muon in response to the antiparticle wave from the electron, not the wave from the muon. But the antiparticle wave from the electron that looks like the negative phase antiparticle wave from the muon will be a positive phase wave. So the anti-KG particle still has positive momentum, causing the muon to recoil when it is emitted and the electron to recoil when it is absorbed.

For a vertex located in the $-k$ direction the opposite occurs. The $\mathrm{KG}$ particle wave from the muon is negative phase, so a negative momentum is transmitted. The emitting muon "recoils negatively," so to speak, as does the absorbing electron. But this is exactly what is needed in the $-k$ direction. The scattering electron always scatters by $k$, regardless of the direction of the vertex from the muon. Similarly, the positive phase KG wave from the muon now corresponds to a negative phase $\mathrm{KG}$ wave from the electron. So the anti-KG particle moving from electron to muon also has negative momentum. In all cases the momentum transfer is correct. This same analysis extends to vertices in all directions around the muon.
Equation (8), however, describes, in current theory, the propagator between two field currents and not between a particle source and another vertex. To obtain the correct mathematical expression for the propagator pictured in Fig. 7, assuming a particle muon source, as required by the elementary waves theory, the propagator must be multiplied by the usual expression for the muon field current and the integral over all possible muon positions performed. The result yields a delta function that provides energy-momentum conservation at the muon vertex. Reverting to photons rather than $\mathrm{KG}$ particles, the electromagnetic wave from the muon is given by

$$
A^{\mu}(x)=\int d^{4} x^{\prime} D_{F}\left(x^{\prime}-x\right) J^{\mu}\left(x^{\prime}\right)
$$

with the electromagnetic propagator given by (Ref. 17, p. 109)

$D_{F}\left(x^{\prime}-x\right)=\int \frac{d^{4} q}{(2 \pi)^{4}} \exp \left[-i q\left(x^{\prime}-x\right)\right] \frac{(-1)}{q^{2}+i \varepsilon}$

(Remember again the reversal of $x$ and $x^{\prime}$ because of the reverse motion of the waves.) Substituting in the usual expression for the Dirac current, as well as the usual expressions for the muon wave functions (Ref. 17, pp. 109, 110), and performing the integral over $d^{4} y$, one obtains

$$
\begin{aligned}
A^{\mu}(x)=\frac{e}{V}( & \left.\frac{m^{2}}{E_{\mathrm{i}} E_{\mathrm{f}}}\right)^{1 / 2} \int d^{4} q \exp (i q \cdot x) \delta(p-p+q) \\
& \times \frac{(-1)}{q^{2}+i \varepsilon} \bar{u}\left(p_{f}\right) \gamma^{\mu} u\left(p_{i}\right)
\end{aligned}
$$

where $p_{\mathrm{i}}$ and $p_{\mathrm{f}}$ are the initial and final momenta of the muon, respectively.

In spite of the delta function, this integral cannot yet be performed. The integral describes the electromagnetic elementary wave that originates from the collection of all possible final muon states: all possible values of $p_{\mathrm{f}}$. So $p_{\mathrm{f}}$ is variable and depends on $q$. Only when this integral is placed in another integral containing the electron current for a specific electron scattering does the integral then select out the particular propagator wave with momentum transfer $q$ for the scattering.

One simply postulates that the interaction of each elementary photon wave with the muon as it passes through the muon is such as to produce Eq. (13) for the electromagnetic field. But, again, this is equivalent to assuming the usual muon "current" form for the vertex for each potential muon scattering.

The scattering of the electron waves caused by this electromagnetic field is given by the usual electron current formula at each vertex. The scattering matrix is then given by

$$
\begin{aligned}
& S_{f i}=-i \int d^{4} x\left\{e \bar{\psi}_{f}(x) \gamma_{\mu} \psi_{i}(x)\right\} A^{\mu}(x) \\
& =-i \int d^{4} x^{\prime} d^{4} x\left\{e \bar{\psi}_{f}(x) \gamma_{\mu} \psi_{i}(x)\right\} J^{\mu}\left(x^{\prime}\right) .
\end{aligned}
$$


Substituting Eq. (13) into (14), again putting in the usual expressions for the electron wave functions, and performing the integrals yields the correct equation for the scattering matrix: using standard notation (Ref. 17, p. 110).

$$
\begin{gathered}
S_{\mathrm{fi}}=\frac{-i e^{2}}{V^{2}}(2 \pi)^{4} \delta^{4}\left(P_{f}-P_{i}+p_{f}-p_{i}\right)\left(\frac{m^{2}}{e_{f} e_{i}}\right)^{1 / 2}\left(\frac{M^{2}}{E_{f} E_{i}}\right)^{1 / 2} \\
\times\left\{\bar{u}\left(p_{f}, s_{f}\right) \gamma_{\mu} u\left(p_{i}, s_{i}\right)\right\} \frac{1}{\left(p_{f}-p_{i}\right)^{2}+i \varepsilon}\left\{\bar{u}\left(P_{f}, S_{f}\right) \gamma^{\mu} u\left(P_{i}, S_{i}\right)\right\},
\end{gathered}
$$

Although the electromagnetic elementary waves emitted by the muon are actually described by Eq. (13) and not by the usual propagator for interacting fields, Eq. (12), the elements of the usual propagator clearly are the guts of expression (13). So the above description of the elementary waves making up the usual propagator clearly carries over to (13).

There is another important physical difference between the elementary waves description of the scattering process and the usual description, and that involves the normalization of the waves. In usual quantum theory normalization is defined in terms of the number of particles per unit volume. But the elementary waves are not particles, and their amplitude does not express a number of particles following them. However, as has now been established, the matrix elements for the elementary waves - the probabilistic relationships between incident and scattered wave are the same as in current quantum theory. So clearly one will get the correct cross section if one simply normalizes the elementary waves in the same manner as the usual quantum waves (assuming one particle per state), followed by computing the cross section from the matrix elements in the usual manner. This simply amounts to defining a particular normalization for the number of (mathematical) flux lines per unit volume in an elementary wave. Stated more simply, the unit of flux is arbitrary. Choosing the normalization in the usual manner simply amounts to the choice of a particular unit of flux.

Also, as part of the same normalization procedure, one must treat the elementary waves as if they existed in a (large) number of discrete states, rather than in a continuum. The waves actually exist in a continuum of momentum states. But one can quantify the flux by defining discrete states over which one then sums. By using periodic boundary conditions in a large box, and then normalizing the resulting states as above, the result is the same as if one normalized to a specific quantity of flux per momentum range per unit solid angle.

Hence, simply by saying that the muon "emits" the usual propagator for all its possible scatterings and that the electron waves scatter in response to that propagator in the usual manner, with particle quanta then exchanged carrying momenta as per the above description, we see how every detail of the propagator from current theory corresponds pictorially - and quantitatively - to an element of the elementary waves theory. The $i_{\varepsilon}$ has its usual significance in fixing the direction of the particle and antiparticle waves. The factor of $1 / p^{2}$ is the Fourier transform of the $1 / r$ dependence (in amplitude) of the elementary waves produced by the radial divergence of the flux lines making up the propagator. Each flux line corresponds to a certain wave intensity, so the intensity goes as $1 / r^{2}$. The amplitude thus goes as $1 / r$. Each frequency in the overall, spherical propagator thus looks exactly like the usual spherical wave, of form $\mathrm{e}^{i k r} / r$.

Because each normalized elementary wave state looks exactly like the corresponding (in reverse) quantum state for a single particle in current theory, because there are equal numbers of different states in the two theories, because the interactions at each vertex are the same, and the "scattered" wave along every line from a vertex looks the same, we have a wave theory that is mathematically identical to current relativistic quantum mechanics.

What was illustrated above for the Klein-Gordon propagator clearly carries over to the propagator for any field. And if the elementary waves theory reproduces the propagators, clearly it reproduces the entire quantum theory.

Remember, again, that proof of mathematical correspondence only requires that the elementary waves scatter as do the usual quantum waves; it makes no difference which path the particles take. The above description of the correspondence is thus over-complicated by the inclusion of the particle processes. But it has now been shown that the particle processes indeed can "mimic" all wave processes, including those that imply negative and off-axis momenta for the propagator particles.

Higher-order graphs can be understood by generalization from the above, following the usual Feynman rules for putting together the mathematics.

Reduced to essentials, the Feynman "heuristic" derivation of his diagrams shows that they can be understood as reflecting the propagation of wave fields along straight lines between vertices. This is exactly what the elementary waves theory yields.

Consider the process pictured in Fig. 9. This is simply an inelastic scattering of an electron from a muon, in which a photon is emitted. The electron wave propagator traveling from A to B is not actually generated at vertex $\mathrm{A}$. It would be a great coincidence indeed if the incident photon wave and electron wave at A would happen to interact close enough to the target muon that the electron propagator would then terminate at a vertex determined by a photon wave from the muon. Rather, the electron

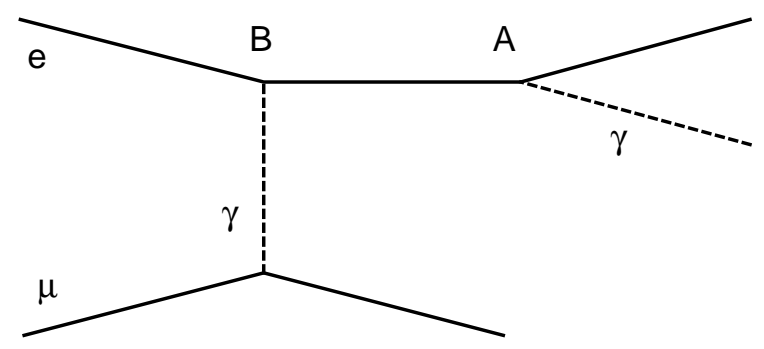

Figure 9. Inelastic electron-muon scattering.

propagator is generated at B. What happens is that the photon propagator wave from the muon generates an electron-positron wave pair at $\mathrm{B}$. The positron propagator consists of positive and 
negative phase velocity parts. When the particle electron arrives, one of two things can happen. Either the positron wave from the electron causes a pair to be generated at $\mathrm{A}$, with the electron from that pair traveling to the detector and the positron traveling to B to annihilate the incident electron; or the incident electron scatters at $\mathrm{B}$ and follows the negative phase positron wave in reverse from $\mathrm{B}$ to A. By "in reverse" here I mean in reverse relative to the usual direction of motion of a particle following the elementary wave object; the particle moves with the wave object, and not in the usual reverse direction. Because the wave is a negative phase velocity wave, the particle moves with the phase waves as it should. We must assume, much as in current theory, that a negative phase wave, traversed in the reverse direction - but not backward in time - looks like an antiparticle wave. In general, a (positive energy) particle can follow a negative phase antiparticle wave in the reverse direction.

But notice that the coherence of the wave toward the source in this example now originates at the target muon and not at the detector. It is the photon wave from the muon that establishes the coherence of the electron wave toward the source. In elastic scattering the coherence originates at the detector and traverses the scattering to then arrive at the source. In inelastic processes the coherence originates at the target.

One can apply this fact about inelastic processes to detectors, this in order to understand how they "emit" waves. A detector is only a detector by virtue of its ability to produce inelastic processes, thus creating an observable effect. So one sees immediately why a detector is the source of coherence among the waves leaving its surface, or at least of those waves corresponding to particle processes that will result in a detection when particles impinge upon it.

Observe that this explanation agrees with the usual quantum statement of the same principle. The usual statement is that processes are mutually coherent - and hence one adds amplitudes unless they are "distinguishable." But "distinguishable" means that the processes produce different observable effects. But different observable effects means a different source for the coherence of the corresponding waves, as just indicated. Hence the wave processes are not mutually coherent.

A summary of the different wave types and the particles that can be stimulated into existence by them is shown in Fig. 10. The first column names the type of wave. In the second column the large arrow identifies the direction of motion of the elementary wave object, the small arrow the direction of motion of the phase wave fronts. Particle waves are shown as a solid line, antiparticle waves as dashed. The third column shows how a particle might move while following the wave, and the fourth column an antiparticle. The sign shown next to each particle arrow is the sign of the momentum

Notice, then, that an opposite phase velocity, antiparticle wave can produce the same particles - moving in reverse - as the particle wave. This statement replaces the usual statement of how anti- (wave-) particles are negative frequency (wave-) particles moving backwards in time. Nothing moves backwards in time in the elementary waves theory, as, of course, must be true in any theory that purports to represent real objects.

A negative phase particle wave traversed in reverse looks like an antiparticle wave. However, it does not become an antiparticle. There is no need in this theory to assume that negative phase waves mysteriously become antiparticles. Why negative phase particle waves traversed in reverse look like antiparticle waves will be explained in a forthcoming paper by the author on the violation of parity, which, as will be demonstrated, is not actually violated at all. (The phenomena occur as described by current parity violation theory, but not because of any fundamental left-right asymmetry of the universe.) But for now this is not postulating anything substantially different from current theory.

The physical interpretation of Feynman diagrams explains how a particle follows its wave. Basically, a particle, say an electron, travels in a straight line as long as it experiences its wave coming from only one direction. When the coherent wave is experienced in a new direction, the electron can then scatter in that new direction. It will do this by emitting or absorbing a particle photon. The photon waves necessary for the emission will always be present, because it is those waves that scattered the electron wave in the first place, thus causing the coherent wave to arrive from the different direction. The photon waves necessary for the absorption are emitted by the electron itself in response to the stimulation of its coherent wave coming from the new direction. If no coherent wave is present when the original wave ends, as in an inelastic scattering, the electron will "jump" into coherence with a new wave, this being determined by the final vertex in the scattering process, as in vertex A in Fig. 9. The probability of a particular "jump" is proportional to the intensity of the new wave.

And as indicated in the previous section, once a particle becomes coherent with a wave, it loses the ability to respond to anything other than that wave. It cannot emit particle photons other than those that will leave it coherent with its wave, because it responds only to the stimulation of its wave in such an emission. And it cannot absorb photons other than those that will leave it coherent with its wave, because it emits only photon waves that correspond to an appropriate scattering, so it can absorb only such photons. Only if the wave is disturbed will the particle leave the wave. 
Wave

Positive
phase velocity
particle

Negative

phase velocity

particle

Positive

phase velocity

anti-particle

Negative

phase velocity

anti-particle
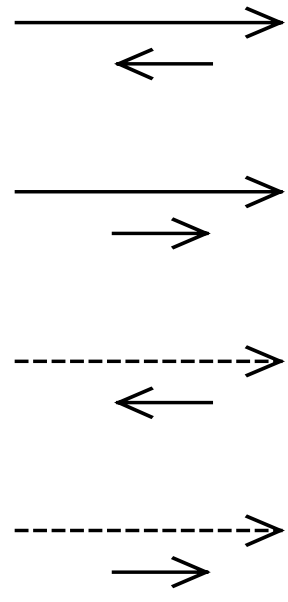

Anti-particle
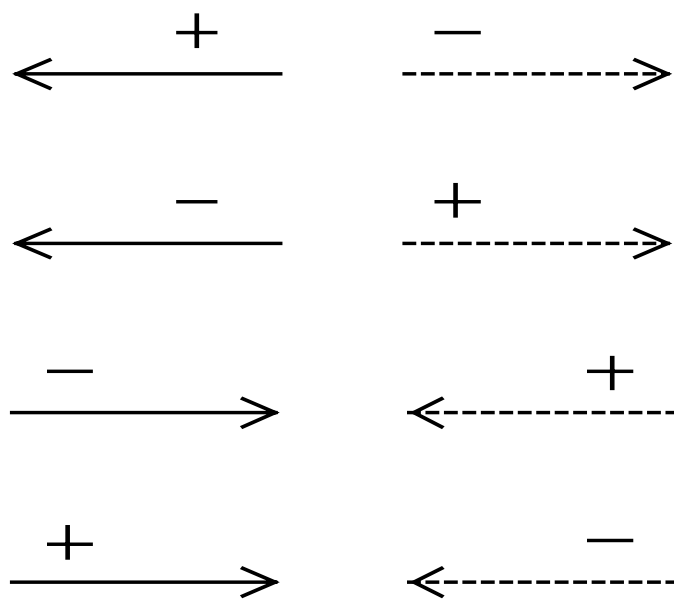

Figure 10. Table of particle types.

Stated more simply, Feynman diagrams picture exactly how a particle follows its wave. Observe, again, that the process is strictly local in nature.

There is some degree of "randomness" in how a particle follows its wave. Internal, "hidden" parameters in a particle determine which of several mutually coherent waves the particle will respond to at a particular vertex. But, as before, the randomness is only apparent, due to ignorance regarding the values of the internal parameters. There is no lack of causality. It will be argued in Sec. 13 that this randomness, resulting as it does from parameters internal to the particles and not from parameters characterizing a particle's motion directly, provides a physical basis for understanding irreversibility in quantum statistical processes.

If a particle is forced to "jump" waves, due to the disappearance of its original wave, one can think of the process as the destruction of the original particle and the creation of a new one following the new wave, as indicated earlier. This is what was meant earlier by saying that the oncoming particle beam was the "source" and that this source was located at the point at which the particles first experience the waves from the target. In a real sense the particle is created at this "source."

This Feynman diagram picture of subatomic processes explains the Aharonov-Bohm effect. ${ }^{(21)}$ As indicated in the previous section, it is the vector potential A that is the primary quantity; it is this that determines the scattering of the electron waves. And those waves can be affected by A even at locations where the particle electron will not scatter, where $\mathrm{E}$ and $\mathrm{B}$ are zero. (This latter is clear from the current mathematical descriptions of A, E, and B.) And it is also clear how an effect on the waves even at locations away from the particle can nonetheless affect the particle's motion, by affecting the probability, at the particle source, that the particle will be created in one state or another. The electric and magnetic fields describe only the direct effect on the particle electron, and not the photon waves themselves that are involved in the underlying electron wave scattering.

The elementary waves theory provides greater physical clarity also in the area of renormalization. Consider "mass renormalization," in which a particle emits and then reabsorbs the same quantum. This means that the elementary wave for the particle interacts with an elementary wave for the quantum that is moving in the same direction as the particle wave and along the same line. And both waves now move with velocity $c$; it is not the case that one moves at $c$ and the other at $v$, the velocity of the particle. So the two waves move along together, interacting again at another vertex. In a real sense the two waves are really one wave compound. So it makes perfect sense to "renormalize" the "bare" wave. In reality a wave is never bare; a bare wave would be the unreal object. Only the "renormalized" object is real.

Because elementary waves exist at all times in all states, there is a continual interaction among the waves even when disorganized, and an organized wave will continually interact with the disorganized background. These are the "renormalization" processes. Space does not permit me to consider them further here.

\section{CLASSICAL MECHANICS AND ELECTRO- DYNAMICS}

The distinction between quantum and classical processes in the elementary waves theory is very much the same as in current quantum theory. In quantum processes the wave that stimulates the emission of a particle, and which the particle then follows, takes multiple paths between "detector" and source. So there is interference between the different waves. The entire space through which the waves travel - and not simply the discrete line along which the particle travels - will then affect the probability of the particle process. In addition, particles have multiple choices when 
they are forced to scatter, due to the fact that coherent waves come at any particular point from multiple directions. In a classical process the wave that stimulates the emission travels from the detector to the emission point over only one path. There is no interference between multipath waves; and the particle must follow only the one path on its return.

Although the wave for a classical process follows only one path, it is actually a sum over a large number of very high-order Feynman graphs, interpreted as in the previous sections. Only very small momentum components of the scattering boson propagator contribute, and one gets, to a close approximation, a curved path for the wave and hence for the particle motion. (Furthermore, it is interference between the various orders of graphs that is responsible for the net repulsion or attraction of charged particles; for individual Feynman graphs there is no net "push" or "pull" on a wave at a vertex, as seen in Sec. 9. At any wave vertex the wave "scatters" with equal amplitude in all directions.)

Because the particle is affected by a wave that traversed only a single path, and because the particle then traverses that same path, one can, for descriptive purposes, leave out the mediation of the wave and treat the process as if the boson field affected the particle directly. The result is classical physics.

The Hamilton-Jacobi formulation of classical mechanics is thus an accurate picture of what is occurring physically. The HamiltonJacobi equation represents the short wavelength limit of the Schrödinger equation. ${ }^{(22)}$ All that needs to be modified from the usual treatment is the direction of the wave, the direction of propagation of the "principle function." It must be reversed.

The basic laws of classical physics - momentum and energy conservation - are, again, primarily laws governing the waves and their interactions at vertices, and only as a secondary consequence are they laws governing the behavior of particles.

Current theory obtains the equations of quantum mechanics by starting with the equations of classical physics and then "quantizing" them, using the canonical quantization procedure. The premise here is that somehow the classical laws are primary, and quantum mechanics is then derived from them. But this has matters backwards. One derives the behavior of larger systems from the behavior of the parts. (Even "emergent" properties can must - be so derived.) In the elementary waves theory the waves simply exist, with their various properties. They are primary constituents of reality. There is thus no need to deduce their behavior from anything. Canonical quantization or the like thus becomes entirely superfluous, as indicated earlier. All that is needed is an understanding of how the classical laws flow from the quantum ones, not the reverse.

Because all electromagnetic "forces" on charged particles are mediated by particle photons - the waves, again, do no "pushing" — it is possible to develop a classical electrodynamics based only on particles, just as classical mechanics can be developed with no Schrödinger waves. This will be the subject of a forthcoming paper by the author.

\section{QUANTUM STATISTICS}

Without giving a complete treatment, one can see physically how the elementary waves theory explains quantum statistics.
Consider the following example.

The quantum-field-theoretic formula for atomic emission of photons includes a factor of $\left(n_{k}+1\right)$, where the $n_{k}$ is the number of photons in the state, which in current theory produce stimulated emission in the usual sense, and the +1 produces the "spontaneous" emission. In the elementary waves theory the +1 corresponds to the elementary wave. The wave is what is there first, before there are any particle photons. The $n_{k}$ must then be the number of emitted particle photons. But the full $\left(n_{k}+1\right)$ factor is involved in the emission probability. It might be asked how the particle photons can induce emission in the same way as the elementary wave, as implied by this formula.

By the same token, when an electron or other fermion is added to a state, no further identical particles can be added. Somehow the presence of the particle in the state prevents the addition of further particles. But if it is the wave that stimulates the addition of a particle, how does the particle offset this effect?

These two situations are, of course, the basis for quantum statistics, Bose and Fermi, respectively.

The answer follows from the results of Sec. 8. A particle emits waves corresponding to all scatterings that it might undergo. This includes scattering by very soft photons, in the case of a charged fermion. So the emitted wave will be the same as the one originally being followed by the fermion. The postulate is that for a fermion this emitted wave is exactly out of phase - but still coherent - with the original wave being followed. So the emitted wave will simply cancel the effect of the original wave in stimulating further particles into the state. Once one particle enters a state, no further particles can join it. This explains the Pauli principle.

Particle photons can interact with other photons to produce particle pairs. So the particle photon must emit the corresponding photon waves. For "soft" interactions the photon will simply reemit the wave it is following, but in this case - for a boson in phase with the original wave. So the amplitude becomes greater, and this leads to the stimulation of more photons into the state.

As a particle follows a wave, it acts to further organize the wave state it is following. Whatever "piece" of a state is being followed, a fermion acts to organize another piece into coherence with the original, but out of phase. For a boson the added piece is in phase.

The fact that it is the wave and not the particle that is responsible for stimulated emission of photons might appear to contradict current theory. But the current theory makes no distinction between the wave and the particle and simply says that the number of "photons", plus 1, gives the appropriate factor. With the elementary waves theory it is clear that the wave does all the stimulating - thus producing both "spontaneous" and "stimulated" emission — while simultaneously the $\left(n_{k}+1\right)$ factor is explained.

Notice, then, that it is actually current theory that is unable to explain why the $n_{k}$ and the +1 both correspond physically to the same thing. The +1 corresponds to no photons, whereas the $n_{k}$ is the number of photons. In the elementary waves theory the physical identity of both terms in the $\left(n_{k}+1\right)$ factor is clear.

Current theory might also seem to be contradicted by the prediction that stimulated emission occurs in a direction opposite to that of the wave. It is well known that a stimulated photon 
travels in the same direction as the stimulating photon. But that is exactly what the elementary waves theory predicts. All particle photons are emitted in a direction opposite to that of the stimulating wave and hence in the same direction as one another.

The usual manner in which the Pauli principle is stated - that a level can be occupied by only one fermion, so any second particle is excluded - implies a nonlocal interaction just as with the decaying atom in a resonant cavity. Only by some nonlocal interaction can the second fermion "know" if the state is available. The elementary waves theory corrects this.

Quantum statistics can thus be understood without the need for any symmetrized or antisymmetrized wave functions. The latter are only required when one assumes that the waves are the particles. Then the waves must reflect the inability of multiple fermions to enter the same state or the enhanced ability in the case of bosons. But in the elementary waves theory the quantum statistical behavior applies only to the particles, not the waves. Just as was found in Sec. 4, there is no need to impose any "entanglements" on elementary waves in order to reproduce multiparticle statistics.

The quantum statistical effects described above will apply only to identical particles. The waves corresponding to different types of particles are always distinguishable and thus cannot act together coherently. We thus have a simple physical explanation for "identical particle" phenomena.

\section{DETAILED QUALITATIVE DESCRIPTION OF ATOMIC EMISSION}

In atomic emission of photons it is well known that the mathematical expression for the emission involves an integration over the entire volume of the atom. And yet the above picture of particle processes maintains that the electron is a pointlike particle and in photon emission emits a pointlike photon. This transition occurs at a single point (actually two or more points are involved, two or more Feynman vertices) as the electron circles in its orbit. So how can this involve an integral over the entire volume?

The answer is that an interaction between the plane electromagnetic elementary waves and the electron elementary waves for the various levels in the atom is occurring at all times and at all points in the volume of the atom. As the result of this interaction, an overall degree of mutual coherence is established between the waves in the two electron levels that might be involved in a jump, through the interaction with the photon wave. The electron, in its orbit, thus experiences the wave of the level into which it might jump as being, to some extent, coherent with itself. Hence at any point it might jump into the lower level, emitting a particle photon in the process. But although this occurs at a single vertex, the degree of coherence that determines the probability at that vertex involves the interaction among the waves over the entire atomic volume.

Although the particle photon is emitted at a single vertex, the electron doesn't jump directly into the lower level at that vertex. Rather, an electron propagator is involved. At the other end of the propagator a second photon is created that travels to the nucleus and causes it to recoil. As far as the electron is concerned, one has, in effect, a single Feynman diagram with the emission of two photons.

Mathematically, again, the results are identical to current theory. Indeed, the problem to be solved in both cases is the same. In current theory and in the elementary waves theory one has a computation of an interaction only among waves, with the aim of determining the degree of "overlap," that is, coherence, between the waves. And the square of the result determines the probability of the particle process.

Notice that the transition rate will not vary from point to point in an electron's orbit. The degree of coherence between the waves of two levels is the same throughout the volume. The "jump" can therefore occur at any point in the electron's orbit.

And the electron does now have an orbit, albeit an orbit that looks like an irregular polygon as it exchanges discrete photons with the nucleus. In all respects we thus have a completely causal, deterministic picture of the atom.

The usual Bohr-theory-like formulation of atomic transitions if looked at again through our prequantum lens — involves a lack of causality. In Bohr theory the energy of an emitted photon is determined by the two energy levels involved. But when an electron begins to emit a photon, how does it "know" what energy to emit until it has already reached the lower level to determine its energy? The electron must first complete the jump in order to know what energy to emit in starting the jump. Or, alternatively, some nonlocal interaction must exist between the nucleus and the electron to tell the electron where the lower level is. But there is no such problem with the elementary waves theory. The waves for both levels exist and interact at all times. And the electron is not required to be located at more than one place at a time in order to understand the involvement of an integral over the entire volume; the waves are located everywhere, not the electron.

The qualitative explanation for the formation of discrete levels in the atom is straightforward. Consider an elementary wave with an energy low enough to correspond to a bound state. And consider one small "piece" of the wave. Starting at a point near the atom, that "piece" will travel around the nucleus, interacting continually with the photon waves from the nucleus. That is, at each vertex in the "orbit" the original free state piece will establish coherence with another free state piece for the next segment, and so on around the nucleus. Upon completing the orbit, coherence is set up with a new "piece" of the original wave. This process then continues as the wave continues to circle the nucleus. Ultimately the entire level becomes mutually coherent. If the various now mutually coherent pieces are in phase with one another, one has an eigenstate; the pieces can act together to stimulate an electron into the state. If the pieces are out of phase, then they cancel each other out as far as stimulation of electrons is concerned. The pieces are still there - the waves do not disappear - but they cannot stimulate any electrons. The net result, as far as any ability to affect particle electrons is concerned, is exactly the levels of current quantum theory.

Level widths occur because a wave with a frequency slightly different from an eigenfrequency can still not suffer total destructive interference, this due to its interaction with other eigenlevels. Again speaking qualitatively, the interaction upsets the wave piece before it has time to circle the atom enough times to render the entire level mutually coherent. The part of the level 
that is mutually coherent will not suffer complete destructive interference. The stronger the interactions affecting a certain level, the less the destructive interference for the slightly off-eigenfrequency wave and hence the greater the width of the level. But a greater interaction means a shorter lifetime for the level. So one can understand, at least qualitatively, the uncertainty relation

$$
{ }_{\Delta} E_{\Delta} t=h
$$

as applied to atomic transitions.

Atomic absorption of a photon follows the same pattern as emission.

A description similar to that given here for the atom also applies to the "jump" of a particle in a box when the box is changed. In current theory the particle is a wave in the box, and when the box is changed the wave jumps into a superposition of waves, each having an amplitude given by the appropriate matrix element. The particle then, mysteriously, appears in only one of those states when one looks at it to determine its state, with a probability for each state given by the square of the matrix element. In the elementary waves theory the particle is following a wave, and when the box is changed there is a matrix element - the same as in current theory - for an overlap between the original wave being followed and each of the new waves in the box. (Actually, it is the wave moving with the particle that is relevant to the jump, as discussed in Sec. 4. However, in a resonant box that is simply the complex conjugate of the wave being followed by the particle, which is exactly what one wants for the matrix element.) This matrix element is determined by integration over the entire volume of the box. The particle jumps immediately into coherence with one of these new waves, with a probability given by the degree of mutual coherence between the old wave and new. But this is given by the square of the very same matrix element. So the probability of observing the particle in one of the new states is mathematically identical to current theory. But now the steps of the process are causal and make sense. There is never any need to assume that the particle was in more than one state at a time. Only the waves are in the multiple states, not the particle.

With this picture of atomic emission and with the understanding of Feynman diagrams from Secs. 8 and 9, one can now understand what was claimed in Sec. 4 regarding an atomic cascade process, namely, that it is a single quantum process and not two separate ones. The waves for each level in an atom exist at all times, with mutual coherence established between pairs of levels as described above. If a particularly strong interaction exists between one pair of levels, a significant portion of the upper level will be coherent with the lower. Then if that upper level is to some degree mutually coherent with a still higher level, an overall coherence is set up between all three levels.

What one has in effect is the Feynman diagram of Fig. 11. Segment 3 is the upper atomic level, 2 the middle, and 1 the lower. (Each level is continually interacting with the nucleus; I have omitted these photons from the diagram.) The wave, moving in the opposite direction, is a single coherent wave starting in the lower level and scattering - through interaction with the incident photon waves - through the middle level to the upper one. When the electron jumps into coherence with this wave in level 3, it is already "destined" to arrive at level 1 simply by following this wave.

Of course, some degree of such "cascade" mutual coherence is set up between all sets of multiple levels at all times, including sets of four and more levels. However, because perturbations cause an electron to jump from one particular coherent "piece" of a level to other pieces in that same level as it circles, a cascade can only happen with a significant probability if the particle remains in the middle level for only a short period of time. Then it follows a single coherent wave throughout the cascade, as pictured in the figure.

The square of the amplitude occurs when the jump of the particle electron into coherence with this overall wave takes place, at some point in level 3. (In general, it is only when a particle jumps into coherence with a new wave that a square, a wave intensity, is involved.) No further square is involved upon emission of either photon, because no jump in coherence of the electron is involved there. However, the emission probability of each photon does still depend on the square of the amplitude of the respective photon wave; the amplitude of each photon wave is one factor in the overall amplitude for the process shown in Fig. 11, so each is squared as part of the square of that amplitude. A factor of $\cos \theta$ appears in the amplitude arising from the angle between the polarization of the two incident photon waves. This is then squared to give the probability that the electron jumps into coherence with this wave during its motion in level 3 . The whole process only occurs if the electron so jumps; and once the jump occurs the rest follows with probability 1 ; so the probability of the initial jump gives the overall probability of the process.

Given this picture of the origin of the $\cos ^{2} \theta$, it is now clear how a (single) delayed choice rotation of either polarizer in the EPR experiment will yield the result predicted by quantum mechanics. If the process occurred in two steps, with the $\cos ^{2} \theta$

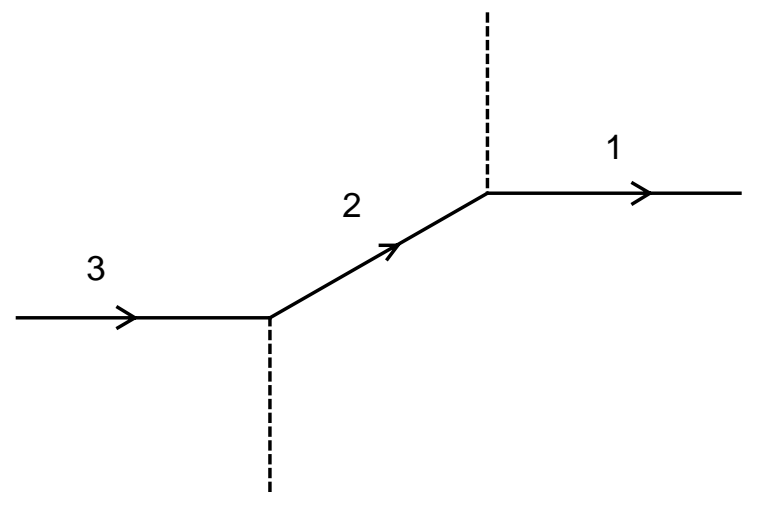

Figure 11. Feynman diagram for cascade emission.

coming only from the emission of the second photon relative to the first, then the explanation for delayed choice would work for the second photon, but would not explain a delayed choice for the first photon.

Figure 11 also makes clear why it is that the photons are accompanied by the (anti-) photon waves, as indicated in Sec. 4 . 
The lines shown for the emitted photons might just as well correspond to absorbed (anti-) photons. That is, the wave interactions for photon absorption occur at the same vertices and are mutually coherent with those for emission. Both interactions are occurring at all times. But these (anti-) photon waves then move in the opposite direction from the waves for the emitted photon, that is, they move with the particle photon when it is emitted.

Clearly, this will be a general property of any diagram. Any vertex that can emit the particle/absorb the particle wave can also, in a mutually coherent manner, absorb the antiparticle/emit the antiparticle wave. That process occurs along with the actual particle emission. So the antiparticle wave travels with the particle and is thus available to participate in the pair process involved in a "jump" of waves (except for slow-moving particles where the wave gets ahead).

Indeed, all vertex processes for particles can be understood as processes of pair creation. At any vertex at which a fermion scatters, by absorbing or emitting a boson, one can think of the fermion process as a "pairs process." The boson creates a pair, the antiparticle of which annihilates the incident fermion, and the particle from the pair then follows the new path of the fermion. And as indicated in Sec. 4, boson emission and absorption can itself be thought of as involving the same process that is involved when a boson "jumps" states, which is itself a pairs process. In all pairs processes the antiparticle is created in response to the antiparticle wave, which is moving along with the incident particle.

In the elementary waves theory it is not necessary, as it is in current quantum mechanics, to relativize the theory in order to obtain pairs. Pair creation is part and parcel of the basic particle dynamics from the outset.

Indeed, one should not have expected anything else. How else could the Dirac equation correctly describe fermion behavior without pairs for energies less than $2 m c^{2}$ and still describe the behavior correctly with pairs for greater energies. Given that the same equation describes the behavior at all energies, the underlying dynamics should be the same at all energies. In the elementary waves theory it is. All vertex processes are simply pairs processes - at all energies. The only difference for energies greater than $2 m c^{2}$ is that permanent pairs can be created in addition to the original particles. But the new pairs are created through exactly the same process that is involved in all particle interactions.

Particle mechanics is thus very simple. Particles move in a straight line from moment of creation to moment of annihilation. Period. Interactions at vertices are all pairs processes, following the interacting waves. That is all there is to it!

\section{IRREVERSIBILITY OF QUANTUM STATISTICAL PROCESSES}

The elementary waves theory provides an explanation for the irreversibility of quantum statistical processes, thus solving another problem not explained, at least to my satisfaction, in current theory.

As indicated in Sec. 10, a classical process is one in which the moving object has only one wave "choice" at each point in its motion. At any point - any vertex - the moving object must move in a particular direction from that point because it is only in that direction that it finds a wave with which it is coherent. All wave processes are reversible; if one reverses a wave, one gets another already existing elementary wave moving in the opposite direction, exactly as in current theory. Reversal of the particle means putting it into coherence with the reversed wave, so it retraces its motion. Classical processes are reversible.

In quantum processes a particle has multiple "choices" at some points along its path. At any point near a target a scattering particle will experience waves from various directions with which it is coherent (all of which originate from the same wave from the detector). So the actual motion of the particle is determined in part by the inner parameters that determine its choice of wave.

But when one reverses the motion of the particle, the inner parameters are not (necessarily) changed. Those inner parameters do not (necessarily) involve motion themselves. So the reversed particle will not necessarily follow the reversed path. Irreversibility occurs whenever the motion of the particle is determined in part by its inner parameters in this manner.

The elementary waves for all processes, classical and quantum, are reversible, just as for the usual Schrödinger waves. But the particle processes in the quantum case are not. Current waveparticle quantum mechanics says that the wave is the particle, which is why the theory is unable to explain irreversibility.

Of course, an individual scattering between two particles, if fully completed without outside interference, is reversible; the particle will always end up following the same wave after the collision regardless of which path it took during the collision. But what if the collision is disturbed by a third particle while it is taking place, as will occur frequently in a gas of colliding particles? The third particle will upset the wave being followed by the original particle. The response of the original particle will depend on exactly where it is located when the disturbance occurs. But that in turn will depend on the particular path chosen up to that point, which in turn depends on the particle's inner parameters. The overall motion is thus no longer simply a function of the initial motion of all the particles, as in classical physics. The inner parameters also play a role. One cannot predict the future motion of a system simply from a knowledge of its initial state of motion.

This is not to say that the behavior is undetermined, but rather only that it is determined by the inner parameters as well as by the initial motion.

The fact that statistical processes are irreversible serves as the evidence indicated earlier supporting the hypothesis that the different wave "pieces" scatter according to different Feynman diagrams. That is, the various order diagrams are not simply terms in a perturbation expansion; they represent how the individual wave "pieces" actually scatter. It is only because of this that the particles have multiple choices at each vertex. If the wave scattering were continuous, then the particles would follow deterministic paths, paths, that is, that would depend only on the prior motion of the particle and not on any internal parameters, which would be reversible. 


\section{GENERAL RELATIVITY}

General relativity should be explainable given the above theory simply by postulating the existence of gravitons and the corresponding elementary waves. All elementary waves interact with the graviton waves in proportion to the mass-energy of the waves. The deflection of a wave at any graviton vertex is very small - so the effect of any graviton particle is very small - and the result is, in effect, a curvature of the otherwise straight-line motion of all waves and therefore of the corresponding particles. This curvature, however, occurs in the framework of a Lorentzian space-time. The result, mathematically, should be exactly Einstein's general theory.

Indeed, the result has to be mathematically the same as Einstein's theory. That theory is deduced by combining special relativity with the equivalence principle. But the above graviton picture, with an interaction strength proportional to the massenergy, leads to the equivalence principle. So one has both special relativity and the equivalence principle. The equations of general relativity follow.

But here also, as with special relativity, there is no need to conclude that space-time is a real "stuff" that is "curved." There is now no contradiction between the view that space is unchanging and yet that Einstein's equations hold. The elementary waves are curved, not the space. One must use a curved geometry, just as one must, in the special theory, use Lorentz transformations. But the curved geometry merely reflects the curved appearance of any coordinates, which are real or imagined real objects. One might describe the space as curved, but by this one merely means that all objects that might be placed in that space are curved.

\section{DISCUSSION}

It has been shown that the elementary waves theory arrives at matrix elements in a manner far simpler than current theory. No canonical quantization is required, nor any second quantization, nor any relativization to obtain pairs, nor any Fock space to understand quantum statistics. One simply has waves, as primary constituents of reality in their own right, along with particles. Because the waves simply exist and propagate as a flux, field equations become superfluous. They are of no value in expressing the fundamental theory.

Indeed, the theory becomes so simple that one can state it without even writing down a differential equation!

It is because of the primary nature of the waves, rather than of field equations, that it was necessary in Secs. 8 and 9 to establish the mathematical equivalence of the elementary waves theory to current quantum mechanics at the level of Feynman diagrams rather than at the level of field equations.

Perhaps it is confusing that I call them "waves" and then state that there are no field equations. But, as described above, they are waves only in the sense that they combine with one another in a wavelike manner when stimulating the emission of particles. There is a periodicity along the "wave." But these are not waves in a medium, and the signal is not carried by wave fronts.

The notion that any periodic "thing" must propagate as the result of a field equation is a carryover from classical physics. One can deduce wave equations for classical waves from the physics of the medium through which the wave propagates. This notion was then applied to quantum waves, even though no medium is involved. But this is erroneous. It is only if the physics of the waves comes from that of the medium that it makes sense to describe the waves primarily in terms of a field equation. The field equation, after all, primarily describes the medium; the waves are the consequence. If the waves are the primary things, without there being any medium, then one simply describes the waves. The field equation becomes physically superfluous.

It might appear as if Maxwell's equations are required to understand classical electromagnetic waves, where there is no medium. Somehow it is the combination of electric and magnetic fields that produces the waves, as Maxwell theory is usually interpreted. However, as shown above, and as will be argued more fully in the forthcoming paper on classical electrodynamics, it is really the (four-dimensional) vector potential that is the primary quantity, as proved, for example, by the Aharonov-Bohm effect. ${ }^{(21)}$ But the field equation for the vector potential is simply the wave equation, and one can fully describe the resulting waves aside from such an equation. Electric and magnetic fields, again, refer to the resulting particle effects.

One of the most "weird" aspects of current quantum theory is precisely the fact that one uses "fields," which, in fact, are nothing in their own right, but which are nonetheless, somehow, the particles. We saw this above with the "available states"; quantum theory treats them as if they were both something and nothing. This makes no sense. If a field is something, then it is something, something in its own right, aside from the particles that "arise" from it. If the field is nothing then it is nothing. And no particles can arise from nothing. Something is something. Nothing is nothing.

So it is not the elementary waves theory that should not be criticized for the fact that it has no field equations. Rather, current theory should be criticized for the fact that it does have such equations, but without the appropriate physical foundation.

Perhaps the elementary "waves" might better be termed "periodic fluxes." Rather than being waves, which might imply a medium, they are fluxes with a wavelike pattern. If one wishes to call the collection of waves an "aether," one might say that the waves are a flux of aether stuff carrying a wavelike pattern, bearing in mind my earlier reservations on the idea of a "stuff" making up the waves.

Because the waves are elementary, it is impossible to describe them except in terms of their interactions at vertices. It is not possible - not philosophically meaningful - to specify the nature of the variable or variables that change in a periodic manner along a wave, other than to say that the effects at the location of particles and vertices reflect this periodic structure. If one has indeed reached the most "elementary" level, one can only describe what exists, not explain it. Explanations always flow from the more elementary to the less.

I have frequently used the term "coherence" in referring to the "organization" that occurs with the waves. Although the meaning here is somewhat different from the usual, I have retained the term because, in its most essential sense, the meaning is the same: mutually coherent waves are combined by adding amplitudes, incoherent by adding intensities. 
In classical wave theory there is no need for any parameter to distinguish mutually coherent waves from other waves in order to explain their behavior. ${ }^{(23)}$ There is no need for mutually incoherent waves to "tell each other apart." However, for the elementary waves some such parameter is necessary. Consider a simple scattering. The wave arriving at the particle source from the target consists of "pieces" resulting from the scattering of waves impinging on the target from all directions. Some pieces are mutually coherent and others are not. How do these pieces, if they are of the same energy and direction of motion, tell one another apart?

Part of the answer was indicated in Sec. 7. Waves must carry parameters characteristic of the frame of reference of the particle that created the "organization" carried by that wave. And waves can only mutually interfere, so one would postulate, if they have the same values of these parameters. Also, perhaps parameters in an organized wave reflect the "history" of the wave, the net angle of scattering since its "emission," say. Mutual coherence might also require equality of these parameters.

This picture itself leaves some problems. So at this point it must simply be said that the waves are able to tell one another apart by some means, the evidence being the enormous quantity of information that is integrated and explained by the resulting picture. How this occurs remains to be explained.

The basic dynamic variables of physics - mass, energy, momentum, etc. - acquire a very different meaning in the elementary waves theory, as indicated earlier. Consider momentum. In classical physics momentum means (roughly speaking) the physical impulse that something can transmit when it runs into another object. It is defined by the action that the entity can exhibit. Or, as this is frequently stated, momentum is a "descriptive" concept; specifically, it describes behavior, but does not explain it.

In the elementary waves theory momentum acquires a clear meaning as a spatiotemporal property of the waves, a property that can be identified (or "pictured") aside from any specific action exhibited by the waves. Momentum is the parameter corresponding, in the usual manner, to the wavelength of the wave. And it is the resulting behavior of the wave - caused by the fact that it has a specific wavelength - that then explains the behavior of particles following that wave.

The elementary waves might be described initially simply in terms of their wavelength and frequency (for scalar waves). The laws of momentum and energy conservation would then be deduced as a product of the laws describing the vertex interactions of the waves.

Of course, one does need to parametrize the vertex processes. And one might correctly say that momentum and energy are conserved at a wave vertex. But clearly the characteristics of the vertex are what now explain the classical laws of momentum and energy conservation, not the reverse. And, again, the momentum and energy can now be defined in terms of the "picture" of the waves, without reference to the resulting behavior. These are attributes of the waves, not a description of their interactions.

It is my prediction that a great deal of further simplification will be possible in this theory. Rather than requiring a different set of waves for particles of different spin, or even of different mass, instead only one set of waves might be required, the components of which can then be placed into various mutually coherent combinations corresponding to the different kinds of particles. Furthermore, this might be the basis for explaining physically why the different kinds of fundamental forces are really the result of a common process, something which, in my judgment, has not been done satisfactorily with the current "standard model," which, if anything, merely complicates things further with more equations. (Most aspects of the results of the standard model, however, should carry over to the elementary waves theory. If Feynman diagrams capture the standard model results, then the translation to the elementary waves theory is exactly as described in Secs. 8 and 9.) The goal should be a physical understanding of the entities involved, not the discovery of a deeper field equation. Reality consists of real objects, not field equations.

\section{CONCLUSIONS}

It has been shown that the elementary waves theory reproduces the predictions of relativistic quantum mechanics in detail. In essence, all that has changed is that the waves go from detector to source, with the square at the source. The mathematical description of the waves is in most instances identical. Multiparticle phenomena involve a much simplified mathematics, but with the same quantitative result. Whatever experimental results are predicted by the wave-particle interpretation are thus predicted equally well by the elementary waves picture, with the exception of double-delayed-choice experiments, where I predict that a corrected experiment will confirm the elementary waves theory and refute current nonlocal quantum mechanics.

But in addition to reproducing the mathematics, the elementary waves theory also answers all the many open questions concerning measurement. These questions have not been answered satisfactorily in the wave-particle theory. Thus based simply on a consideration of the degree to which the theory can account for all quantum phenomena in a consistent manner, the elementary waves theory is clearly superior.

But, more importantly, the elementary waves theory is both local and deterministic. All the principal "weird" aspects of quantum mechanics are explained with a picture in which all objects have an exact state at all times, and in which all causal relationships are local, deterministic, and in the proper time sequence. This is accomplished in a manner that does not conflict with Bell's theorem.

In the elementary waves theory all aspects of the mathematics of quantum mechanics correspond to something real. There are no formulas whose only real referents are "dial readings" and their relationships. Any dial readings are measurements of real properties or behavior of real entities.

In no way are we required to conclude that there is a breach between the real and the observed, between our knowledge and the objects of that knowledge. What we see is what exists. A measuring apparatus affects the creation of the particles that are observed; but this does not require the conclusion that a particle lacks identity until actually detected.

It has been clearly demonstrated that major aspects of the current wave-particle theory are interpretative, that is, that one 
cannot conclude that they are true simply because the formulas yield the correct answer. Physical assumptions - specifically the forward motion of the waves - have been made in the present theory that are not dictated by the (matrix element level) mathematics. This is proved by the fact that another theory, based on different hypotheses, reproduces the same mathematics. If the wave-particle picture were part and parcel of the mathematics of quantum mechanics, then any theory that reproduced that mathematics would necessarily simultaneously reproduce the wave-particle picture. But the elementary waves theory does not do this. Clearly, then, the wave-particle picture is interpretative and not simply descriptive.

If one wishes to validate the wave-particle theory, it is thus not enough simply to say that the formulas work. One must also be able to demonstrate, based on empirical evidence, that the further physical hypotheses are correct. Specifically, one must be able to demonstrate that the waves move forward and not in reverse.

But this cannot be done for the wave-particle theory. The test of any hypothesis is this: does the hypothesis fit all the facts with no contradictions? Bell ${ }^{(1)}$ has shown - although he was not aware of this - that the forward-wave theory fails this test. If one accepts the forward-wave picture of things, one is forced to accept the existence of nonlocal behavior: interactions over long distances by no physical means. But an interaction by no means is a contradiction. Bell's theorem thus constitutes a reductio ad absurdum of the forward-wave interpretation. Far from validating current quantum theory and its associated philosophy, Bell's theorem proves it incorrect.

Indeed, all the "weird" conclusions of current quantum mechanics constitute refutations of the forward-wave theory. The very idea of "wave-particles" is self-contradictory. Whether it be particles located many places at the same time or cats that are neither dead nor alive, or what have you, these are all contradictions. Many physicists have become used to the idea that such contradictions must simply be lived with; but it is now clear that that is not the case. One can understand quantum mechanics, that is, the mathematics of quantum mechanics, without these contradictions. We do not live in a weird universe.

A theory that results in absurd conclusions is false. Reality does not make mistakes; only physicists do.

Previous attempts to interpret quantum mechanics, whether it be the Copenhagen interpretation, de Broglie's pilot waves, Bohm's quantum potential, or what have you, have all failed for the same simple reason: they all had the waves moving in the wrong direction. Once this error was made, the nonlocality was locked in. There was no way around it.

This is why the various "hidden-variable proofs" worked. By proving that wave-particle quantum mechanics could not be "fixed" through the addition of new variables, these proofs simply demonstrated that an error had already been made and that the weird results of quantum mechanics could not be "fixed" until that error was corrected.

The elementary waves theory provides a real explanation of quantum phenomena. The wave-particle theory, on the other hand, is actually not a theory at all. It is an antitheory. It says, in effect, that an explanation is impossible, that quantum phenomena are inherently contradictory and therefore not rationally understandable. But in fact these phenomena are not inherently contradictory. They are understandable.

Most of what has been accomplished above follows simply from the recognition that the waves move in reverse. With that one small change

(1) we have explained the double-slit experiment without requiring that a particle go through both slits;

(2) we have resolved the EPR paradox, obtaining a local understanding of Bell's theorem;

(3) we have resolved the Schrödinger cat paradox;

(4) we have given a pictorial, deterministic explanation for the uncertainly principle;

(5) we have explained the Pauli principle;

(6) we have given a simple, picturable explanation for quantum statistics more generally, Bose and Fermi;

(7) we have explained the mysterious phenomena involving noncommuting observables;

(8) we have explained the constancy of the velocity of light relative to all observers on simple, physical grounds, thereby explaining the special theory of relativity;

(9) we have explained, in principle, the general theory of relativity;

(10) we have explained the Aharonov-Bohm effect, this in a local manner;

(11) we have shown how in principle one can understand the irreversibility of quantum statistical processes;

(12) we have shown that one can understand Feynman diagrams in a simple, pictorial manner;

(13) we have eliminated all the mysteries and unanswered questions concerning quantum measurement;

(14) we have obtained a fully picturable understanding of atomic physics.

Virtually all the principle mysteries of twentieth-century physics disappear as soon as one corrects the one error.

Furthermore, the theory accomplishes what has been sought for many years: a single framework within which all known physical phenomena can be comprehended. Specifically, gravitational phenomena can now be understood as part of the same structure that explains electromagnetic, weak, and strong interactions.

And all of this is accomplished with a theory that is far simpler than current quantum mechanics. Given the fact that the elementary waves theory arrives at the correct equations without canonical quantization, without second quantization, without relativization, without wave entanglements, etc., clearly those who would continue to uphold current quantum theory have their work cut out for them. They must justify the continued inclusion of all these now superfluous aspects of the theory. Occam's razor does not say that the simpler theory is correct. But it does say that one cannot validly add further aspects to a theory without additional evidence, evidence going beyond what is explained by the simpler theory.

Clearly, the fact of reverse elementary waves has been demonstrated. Too many things are explained by the one simple hypothesis to conclude otherwise. 


\begin{abstract}
Acknowledgment
I would like to acknowledge the numerous suggestions and continual support of Dr. Harry Binswanger during the writing of this paper. I am indebted to Mr. M.A. Titko for useful discussions and corrections of several errors. My thanks to Mr. William Dixon for his encouragement.
\end{abstract}

Received 22 April 1994.

\begin{abstract}
Résumé
Une erreur a été trouvée dans la théorie quantique fondamentale. On montre que cette erreur est la source de divers aspects non causals de la théorie. Lorsque cette erreur est corrigée, une nouvelle théorie apparaît qui est à la fois locale et déterministe, mais néanmoins n'entre pas en conflit avec le théorème de Bell. La nouvelle théorie retrouve quantitativement toutes les prédictions courantes de la mécanique quantique à l'exception du double choix retardé du phénomène d'Einstein-Podolsky-Rosen. Les imperfections des expériences d'Aspect sont soulignées et une expérience définitive est proposée. Le paradoxe d'Einstein-Podolsky-Rosen est résolu. Le principe d'incertitude est dérivé sur une base causale et déterministe. La théorie est essentiellement relativiste, c'est-à-dire la constante de la vitesse de la lumière c relative à tout observateur apparaît comme une conséquence immédiate de la nouvelle théorie. La relativité générale prend donc une interprétation physique simple. Le diagramme de Feynman prend la forme d'une illustration. Cette théorie fournie une explication physique claire de l'effet Aharonov-Bohm et suggère une explication au procédé quantique statistique irréversible. La mécanique statistique de Bose et de Fermi est expliquée d'une façon simple et illustrative. Enfin, il est démontré que les phénomènes quantiques et relativistes peuvent être compris d'une façon objective dans laquelle les faits sont les faits, la causalité est valide et la réalité est réelle. La théorie fournie un cadre simple dans lequel tous les phénomènes physiques simples peuvent être compris réalisant ainsi l'objectif de la théorie d'un champ unifié.
\end{abstract}

\section{References}

1. J.S. Bell, Physics 1, 195 (1964).

2. S.J. Freedman and J.F. Clauser, Phys. Rev. Lett. 28, 938 (1972).

3. M. Lamehi-Rachti and W. Mittig, Phys. Rev. D 14, 2543 (1976).

4. A. Einstein, B. Podolsky, and N. Rosen, Phys. Rev. 47, 777 (1935).

5. A. Aspect, J. Dalibard, and G. Roger, Phys. Rev. Lett. 49, 1804 (1982).

6. D. Bohm, Phys. Rev. 85, 166 (1952).

7. S. Haroche and D. Kleppner, Phys. Today (Jan), 24 (1989).

8. J.F. Clauser, M.A. Horne, A. Shimony, and R.A. Holt, Phys. Rev. Lett. 23, 880 (1969).

9. J.M. Blatt and V.F. Weisskopf, Theoretical Nuclear Physics (Wiley, NY, 1952), p. 528.

10. R.P. Feynman, Proceedings from the Second Berkeley Symposium on Mathematical Statistics and Probability (University of California Press, Berkeley, 1951).

11. E. Schrödinger, Naturwissenschaften 23, 807 (1935).

12. H. Kaiser, R. Clothier, and S.A. Werner, Phys. Rev. A 45, 31 (1992).

13. S.A. Werner, Phys. Today (Dec), 24 (1980).

14. D.C. Lindberg, Theories of Vision from Al-Kindi to Kepler (University of Chicago Press, 1976).

15. J.D. Jackson, Classical Electrodynamics (Wiley, NY, 1962).
16. W.K.H. Panofsky and M. Phillips, Classical Electricity and Magnetism (Addison-Wesley, Reading, MA, 1962), Chap. 16.

17. J.D. Bjorken and S.D. Drell, Relativistic Quantum Mechanics (McGraw-Hill, NY, 1964).

18. L.D. Landau and R.E. Peierls, Z. Phys. 69, 56 (1931).

19. R. Weingard, "Virtual Particles and the Interpretation of Quantum Field Theory," in H.R. Brown and R. Harre, Philosophical Foundations of Quantum Field Theory (Oxford, 1988), p. 43.

20. J.D. Bjorken and S.D. Drell, Relativistic Quantum Fields (McGraw-Hill, NY, 1965), p. 42.

21. Y. Aharonov and D. Bohm, Phys. Rev. 115, 485 (1959).

22. H. Goldstein, Classical Mechanics (Addison-Wesley, Reading, MA, 1959), Chap. 9.

23. R. Loudon, The Quantum Theory of Light, 2nd ed. (Oxford, 1983).

\section{Lewis E. Little}

101 West Hampton Rd.

Philadelphia, Pennsylvania 19118 U.S.A. 